\title{
The Grothendieck-Riemann-Roch Theorem for Group Scheme Actions
}

\author{
BERNHARD KÖCK \\ Mathematisches Institut II der \\ Universität Karlsruhe \\ D-76128 Karlsruhe \\ Germany \\ e-mail: Bernhard.Koeck@math.uni-karlsruhe.de
}




\section{Contents}

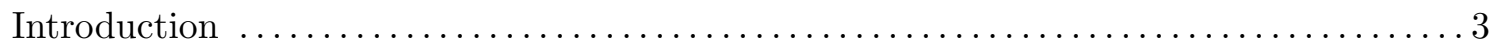

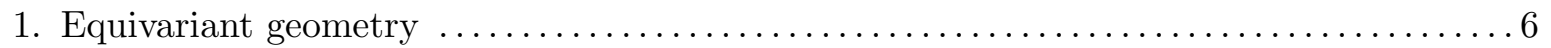

2. The $\lambda$-structure on the equivariant algebraic $K$-theory $\ldots \ldots \ldots \ldots \ldots \ldots \ldots \ldots$

3. The excess intersection formula for $G$-projective local complete intersection

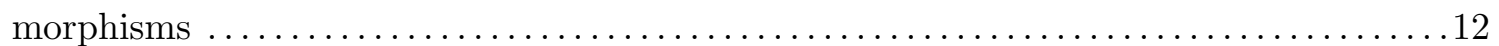

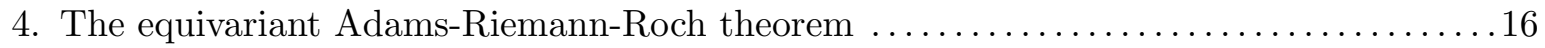

5. The equivariant Grothendieck-Riemann-Roch theorem $\ldots \ldots \ldots \ldots \ldots \ldots \ldots \ldots \ldots \ldots \ldots \ldots$

6. An induction formula for (integral) representations of finite groups $\ldots \ldots \ldots \ldots \ldots \ldots 27$

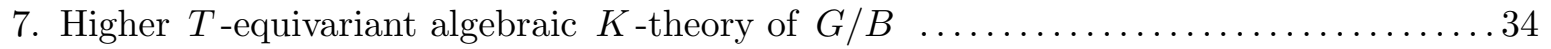

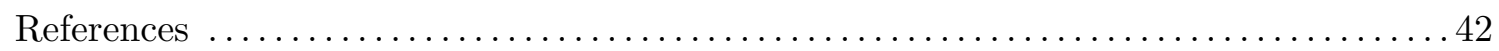




\section{Introduction}

For a projective morphism $f: Y \rightarrow X$ between complex nonsingular varieties, there are essentially two different Riemann-Roch formulas both of which imply the famous HirzebruchRiemann-Roch theorem. The first one says that the canonical map from algebraic to topological $K$-theory commutes with push-forward $f_{*}$. A generalization of this formula to higher $K$-theory of group scheme actions with values in the equivariant étale-topological $K$-theory was given by Thomason in [Th2]. The second one is the Grothendieck-Riemann-Roch formula with values, say, in the graded object associated with the Grothendieck filtration on the algebraic $K$-theory. It describes the behaviour of the Chern character with respect to push-forward. This paper deals with a generalization of the latter formula to the equivariant case, again in the context of higher $K$-theory of group scheme actions.

Let $G / S$ be a flat group scheme. For a $G$-scheme $X / S$, let $K_{q}(G, X)$ denote the $q$-th equivariant $K$-group of $X$, i. e. Quillen's $q$-th $K$-group associated with the exact category of locally free $G$-modules on $X$, and let

$$
K(G, X):=\underset{q \geq 0}{\oplus} K_{q}(G, X) .
$$

Then exterior power operations make the Grothendieck ring $K_{0}(G, X)$ a so-called (special) $\lambda$ ring. Applying Grayson's construction of exterior power operations on higher $K$-theory, we also obtain maps $\lambda^{i}, i \geq 0$, on $K_{q}(G, X), q \geq 1$ (see section 2). Conjecturally, $K(G, X)$ together with these maps is a (special) $\lambda$-ring. Whereas two of the three axioms of a $\lambda$-structure are rather easy to verify (see section 2), so far no proof is known for the remaining axiom concerning the composition of exterior power operations. Apart from Grassmann varieties (see section 2) and generalized flag varieties $G / B$ (see section 7 ), this axiom can be checked in the rather general situation, when $X$ is nonsingular and $G$ is a finite constant group scheme whose order is invertible on $X$ (see [Ko2] and section 2).

Now let $f: Y \rightarrow X$ be a $G$-projective local complete intersection morphism between $G$-schemes $Y$ and $X$. We furthermore assume that each coherent $G$-module on $X$ (and then also on $Y$ ) is a $G$-quotient of a locally free $G$-module (of finite rank). This assumption holds in most interesting cases by Thomason's work [Th3]. Then the association

$$
\mathcal{F} \mapsto \sum_{i \geq 0}(-1)^{i}\left[R^{i} f_{*} \mathcal{F}\right]
$$

$(\mathcal{F}$ a locally free $G$-module on $Y$ ) "induces" a push-forward homomorphism

$$
f_{*}: K(G, Y) \rightarrow K(G, X),
$$

the so-called equivariant Euler characteristic or Lefschetz trace (see section 3). The equivariant Riemann-Roch problem is to compute this Euler characteristic $f_{*}$.

Our first answer to this problem is a formula for the behaviour of the Adams operations $\psi^{j}$, $j \geq 1$, with respect to $f_{*}$ (see section 4). For this, let $\hat{K}(G, X)\left[j^{-1}\right]$ be the completion of $K(G, X)\left[j^{-1}\right]$ with respect to the Grothendieck filtration on $K(G, X)\left[j^{-1}\right]$. (It would also suffice to complete with respect to the $I$-adic filtration where $I$ is the augmentation ideal in $K_{0}(G, X)$.) Let $\theta^{j}(f) \in K_{0}(G, Y)$ be the $j$-th equivariant Bott element associated with $f$.

Theorem (Equivariant Adams-Riemann-Roch formula). For all $y \in K(G, Y)$ and $j \geq 1$, we have

$$
\psi^{j} f_{*}(y)=f_{*}\left(\theta^{j}(f)^{-1} \cdot \psi^{j}(y)\right) \quad \text { in } \quad \hat{K}(G, X)\left[j^{-1}\right] .
$$


Apart from the generalization from finite constant group schemes to (more or less) arbitrary flat group schemes, the essential improvement of this formula (compared with the formulas in my previous paper [Ko2]) is that it drops the assumption that either $G$ acts trivially on $X$ and $Y$ or that $f$ is a regular closed $G$-immersion. However, this improvement makes it necessary to complete $K$-theory as defined above in order to be able to invert Bott's element (see section 4).

Our second answer to the equivariant Riemann-Roch problem is a formula for the behaviour of the Chern character

$$
\operatorname{ch}: K(G, X) \rightarrow \hat{\operatorname{Gr}} K(G, X)_{\mathbb{Q}}=\prod_{n \geq 0} F^{n} K(G, X)_{\mathbb{Q}} / F^{n+1} K(G, X)_{\mathbb{Q}}
$$

with respect to the equivariant Euler characteristic $f_{*}$ (see section 5 ): Whereas the equivariant Adams-Riemann-Roch theorem does not use the third axiom of a $\lambda$-structure, we now assume not only the conjecture mentioned above (concerning the $\lambda$-structure on higher $K$-theory) but also the following conjecture (concerning Grothendieck groups as well) to be true:

Conjecture: The equivariant Euler characteristic $f_{*}: K(G, Y) \rightarrow K(G, X)$ is continuous with respect to the Grothendieck filtrations, i. e. $f_{*}$ induces a homomorphism

$$
\hat{f}_{*}: \hat{K}(G, Y) \rightarrow \hat{K}(G, X)
$$

between the completions.

Let $\operatorname{Td}(f) \in \hat{\operatorname{Gr}} K(G, Y)_{\mathbb{Q}}$ denote the equivariant Todd class associated with $f$.

Theorem (Equivariant Grothendieck-Riemann-Roch formula). Under the above assumptions, $\left(\hat{f}_{*}\right)_{\mathbb{Q}}$ respects the Grothendieck filtrations up to a shift, and for all $y \in K(G, Y)$, we have

$$
\operatorname{ch}\left(f_{*}(y)\right)=\hat{\operatorname{Gr}}\left(f_{*}\right)_{\mathbb{Q}}(\operatorname{Td}(f) \cdot \operatorname{ch}(y)) \quad \text { in } \quad \hat{\operatorname{Gr}} K(G, X)_{\mathbb{Q}} .
$$

This theorem is proved in section 5 where we also present some special cases (see below) supporting the above conjecture on $f_{*}$. In the non-equivariant case, this theorem was proved by Grothendieck (see [SGA6]) for $K_{0}$-groups and by Soulé (see [So]) for higher $K$-groups.

In section 6, we apply the equivariant Adams-Riemann-Roch theorem to the following situation. Let $H$ be a subgroup of the finite (abstract) group $G, A$ a commutative ring, and let $i_{*}$ : $K(H, A) \rightarrow K(G, A)$ be the induction map.

Corollary (Induction formula). For all $x \in K_{0}(H, A)$ and $j \geq 1$, we have

$$
\psi^{j} i_{*}(x)=i_{*} \psi^{j}(x) \quad \text { in } \quad \hat{K}(G, A)\left[j^{-1}\right] .
$$

This formula for instance implies that the induced representation $A[G / H]$ viewed as an element of $\hat{K}_{0}(G, A)\left[j^{-1}\right]$ is invariant under the Adams operations $\psi^{j}, j \geq 1$. Whereas this fact can directly be checked for $A=\mathbb{C}$ by identifying representations with characters and by using Atiyah's computation of the kernel of the completion map, I do not know an elementary proof of this fact for $A=\mathbb{Z}$ (but see Remark (6.10) for related formulas). An interesting application of this fact is the construction (see section 6) of universal annihilators for the Chern classes of the induced representations $A[G / H]$ in the sense of Grothendieck (see [Gro2]) or Thomas (see [Tho]). In the case $A=\mathbb{C}$, we furthermore interpret a deep theorem of Atiyah (see [At]) as an example where $f_{*}$ is continuous as conjectured above.

Using some ideas from the paper [KK2] of Kostant and Kumar, we compute the higher $T$ equivariant (algebraic) $K$-theory of $G / B$ in section 7 . Here, $G$ is a simply connected split 
semisimple group scheme over a regular base $S, T$ a maximal split torus in $G$, and $B$ a Borel subgroup of $G$ containing $T$. We deduce from this computation that $K(T, G / B)$ is a (special) $\lambda$-ring and that the push-forward homomorphism $\pi_{*}: K(T, G / B) \rightarrow K(T, S)$ associated with the structure morphism $\pi: G / B \rightarrow S$ is continuous as conjectured above. Applying the equivariant Grothendieck-Riemann-Roch theorem to $\pi$, we finally give a new proof of the famous Weyl character formula.

We now describe further relations connecting the subject of this paper with several rather different areas.

Chinburg, Erez, Pappas, and Taylor have independently proved similar equivariant RiemannRoch formulas for arithmetic schemes with a finite group action (see [CEPT]).

Current research is concerned with the question how to express the "Adams operations" defined by Cassou-Noguès and Taylor on locally free classgroups (see [CNT]) in terms of (exterior) power operations (see [BC], [Ko8], and [Ko9]). In the paper [BC], Burns and Chinburg establish a formula for these "Adams operations" for certain ambiguous ideals in a tame Galois extension of a number field. In the paper [Ko9], we give an algebro-geometric explanation of their formula using the equivariant Adams-Riemann-Roch theorem of this paper.

Edidin and Graham develop an equivariant intersection theory in their paper [EG]. They establish a Riemann-Roch isomorphism between equivariant $K$-theory and equivariant intersection theory and they prove a version of the continuity conjecture mentioned above for arbitrary reductive groups acting on smooth varieties over a field.

In the paper $[\mathrm{BV}]$, Brion and Vergne prove an equivariant Riemann-Roch theorem for complete, simplicial toric varieties. One should be able to deduce their theorem from the equivariant Grothendieck-Riemann-Roch theorem of this paper. This would in particular solve the problem mentioned at the end of the paper $[\mathrm{Mo}]$ by Morelli. I hope to say more on this in a future paper.

Finally, I would like to mention that several people working in Arakelov theory expect that there exist theorems of Riemann-Roch type in equivariant Arakelov $K$-theory which would be analogues or generalizations of the results of this paper.

Since probably facts and proofs concerning algebraic geometry, (higher) $K$-theory, group schemes, (equivariant) Riemann-Roch theory, etc. are sometimes assumed to be well-known without giving a reference in the text, I now give a list of books which I have used and where these facts and proofs are likely to be found: [EGA], [FL], [Ha], [J], [Ko0], [Q], [SGA3], [SGA6].

This paper is a revised version of my "Habilitationsschrift" ([Ko6]). I would like to thank T. Chinburg, F. Herrlich, W. v. d. Kallen, I. Panin, D. Roessler, C. Soulé, G. Tamme, M. J. Taylor, and R. W. Thomason for their encouraging interest in this project and for discussing several questions during the preparation of this paper. Especially, I would like to thank C.-G. Schmidt for his support in so many ways during the last years. 


\section{Equivariant Geometry}

The purpose of this section is to introduce some notations used throughout this paper and to recall some facts and constructions of equivariant geometry for the reader's convenience.

Let $S$ be a noetherian scheme, and let $G$ be a flat group scheme over $S$. All fibred products of schemes without further specifications will be taken over $S$. A $G$-scheme over $S$ is an $S$ scheme $X$ together with an $S$-morphism $m_{X}: G \times X \rightarrow X$ which satisfies the usual associativity property. For any $G$-schemes $X, Y$ over $S$, the set of $G$-equivariant $S$-morphisms (for short: $G$-morphisms) from $X$ to $Y$ is denoted by $\operatorname{Mor}_{G}(X, Y)$. We denote the category of $G$-schemes of finite type over $S$ by $(G$-schemes $/ S)$.

Let $X \in(G$-schemes $/ S)$. The following definition describes the same as Mumford's notion "G-linearization" (see Chapter I, $\S 3$ of [Mum]).

(1.1) Definition. A $G$-module on $X$ is an $\mathcal{O}_{X}$-module $\mathcal{M}$ together with an isomorphism

$$
m_{\mathcal{M}}: m_{X}^{*} \mathcal{M} \stackrel{\sim}{\rightarrow} \operatorname{pr}_{X}^{*} \mathcal{M}
$$

of $\mathcal{O}_{G \times X}$-modules which satisfies the following associativity property:

$$
\left(\operatorname{pr}_{2,3}^{*} m_{\mathcal{M}}\right) \circ\left(\left(1 \times m_{X}\right)^{*} m_{\mathcal{M}}\right)=\left(m_{G} \times 1\right)^{*} m_{\mathcal{M}} .
$$

Here, $m_{G}$ denotes the multiplication $G \times G \rightarrow G$, and $\operatorname{pr}_{X}: G \times X \rightarrow X$ and $\operatorname{pr}_{2,3}$ : $G \times G \times X \rightarrow G \times X$ denote the obvious projections. A homomorphism of $G$-modules on $X$ is a homomorphism of the underlying $\mathcal{O}_{X}$-modules which is compatible with the $G$-structures. We denote the category of $G$-modules on $X$ by $\mathcal{N}(G, X)$. The full subcategory of $\mathcal{N}(G, X)$ consisting of coherent modules (respectively locally free modules of finite rank) is denoted by $\mathcal{M}(G, X)$ (respectively $\mathcal{P}(G, X))$.

\section{(1.2) Example.}

(a) The structure sheaf $\mathcal{O}_{X}$ is a $G$-module on $X$ via the canonical isomorphisms $m_{X}^{*} \mathcal{O}_{X} \cong$ $\mathcal{O}_{G \times X} \cong \operatorname{pr}_{X}^{*} \mathcal{O}_{X}$. A closed subscheme $Y$ of $X$ is a $G$-subscheme if and only if the associated ideal sheaf is a $G$-submodule of $\mathcal{O}_{X}$.

(b) Direct sums, tensor products, exterior and symmetric powers, and the dual of $G$-modules on $X$ are again $G$-modules on $X$; they satisfy the obvious universal properties in $\mathcal{N}(G, X)$.

(c) For any $G$-morphism $f: X \rightarrow Y$ in $(G$-schemes $/ S)$, the module of relative differentials $\Omega_{X / Y}$ is a $G$-module on $X$. The direct image and the inverse image define adjoint functors between $\mathcal{N}(G, X)$ and $\mathcal{N}(G, Y)$ : The action of $G$ on the direct image is based on the base change isomorphism (cf. Corollaire (9.3.3) of [EGA] I). The projection formula holds for $G$ modules.

(d) If $G$ is a constant group scheme, i. e. if $G=\coprod_{\gamma \in \Gamma} S$ with an abstract group $\Gamma$, then a $G$-module on $X$ is the same as an $\mathcal{O}_{X}$-module $\mathcal{M}$ together with isomorphisms $\gamma^{*} \mathcal{M} \rightarrow \mathcal{M}$, $\gamma \in \Gamma$, which satisfy the usual associativity properties (cf. section (1.1) of [Ko2]).

(e) A quasi-coherent $G$-module on the base $S$ is the same as a quasi-coherent $\mathcal{O}_{S}$-module $\mathcal{E}$ together with a homomorphism $G \rightarrow \operatorname{Aut}(\mathcal{E})$ of group schemes over $S$ (see Proposition (9.6.4) of [EGA] I for the definition of $\operatorname{Aut}(\mathcal{E}))$.

Proof. Straightforward.

(1.3) Lemma. The categories $\mathcal{N}(G, X)$ and $\mathcal{M}(G, X)$ are abelian categories. The category $\mathcal{P}(G, X)$ is an exact category in the sense of Quillen (see [Q]). A sequence of $G$-modules on $X$ is exact if and only if the underlying sequence of $\mathcal{O}_{X}$-modules is exact. 
Proof. This follows from the flatness of $G$ over $S$.

For any quasi-coherent $G$-module $\mathcal{E}$ on $X$, the associated projective space bundle $\mathbb{P}_{X}(\mathcal{E})$ is a $G$-scheme over $S$ which satisfies the corresponding universal property in $(G$-schemes $/ S)$. Though this fact is well-known, I don't know a reference for it. Since, on the other hand, the projective space bundle is a fundamental tool in the equivariant Riemann-Roch theory, we will prove it here.

For this, more generally, let $F:($ Schemes $/ X) \rightarrow$ Sets be a representable contravariant functor, and let $(\pi: \mathbb{P} \rightarrow X, 1 \in F(\mathbb{P} \rightarrow X))$ be the representing pair. By composing with the projection $\operatorname{pr}_{X}$ and the multiplication $m_{X}$, we obtain functors

$$
F_{\operatorname{pr}_{X}} \text { and } F_{m_{X}}:(\text { Schemes } / G \times X) \rightarrow \text { Sets. }
$$

Let

$$
m: F_{\mathrm{pr}_{X}} \stackrel{\sim}{\rightarrow} F_{m_{X}}
$$

be an isomorphism of functors which satisfies the associativity property

$$
m_{1 \times m_{X}} \circ m_{\mathrm{pr}_{2,3}}=m_{m_{G} \times 1}:\left(F_{\mathrm{pr}_{X}}\right)_{m_{G} \times 1} \rightarrow\left(F_{m_{X}}\right)_{m_{G} \times 1} .
$$

(1.4) Lemma.

(a) There is a unique action $m_{\mathbb{P}}: G \times \mathbb{P} \rightarrow \mathbb{P}$ of $G$ on $\mathbb{P}$ such that the diagram

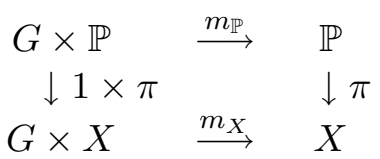

commutes and such that $F\left(m_{\mathbb{P}}\right)(1)=m(G \times \mathbb{P} \stackrel{1 \times \pi}{\longrightarrow} G \times X) F\left(\operatorname{pr}_{\mathbb{P}}\right)(1)$ in $F(G \times \mathbb{P} \stackrel{1 \times \pi}{\longrightarrow}$ $\left.G \times X \stackrel{m_{X}}{\longrightarrow} X\right)=F_{m_{X}}(G \times \mathbb{P} \stackrel{1 \times \pi}{\longrightarrow} G \times X)$.

(b) The pair $(\mathbb{P}, 1)$ equipped with the $G$-action of (a) represents the functor

$$
\begin{aligned}
(G \text {-schemes } / X) \rightarrow & \text { Sets } \\
(T \rightarrow X) \mapsto & \left\{\alpha \in F(T \rightarrow X): F\left(m_{T}\right)(\alpha)=m(G \times T \rightarrow G \times X) F\left(p_{T}\right)(1)\right. \\
& \left.\quad \operatorname{in} F\left(G \times T \rightarrow G \times X \stackrel{m_{X}}{\rightarrow} X\right)=F_{m_{X}}(G \times T \rightarrow G \times X)\right\} .
\end{aligned}
$$

Proof. The uniqueness assertion in (a) is clear. Since $G \times \mathbb{P}$ is the fibred product of $G \times X$ with $\mathbb{P}$ over $X$ with respect to the canonical projections, the $G \times X$-scheme $G \times \mathbb{P} \stackrel{1 \times \pi}{\longrightarrow} G \times X$ represents the functor $F_{\mathrm{pr}_{X}}$. The corresponding canonical element is $F\left(\operatorname{pr}_{\mathbb{P}}\right)(1) \in F\left(G \times \mathbb{P} \stackrel{\mathrm{pr}_{\mathbb{P}}}{\longrightarrow}\right.$ $\mathbb{P} \stackrel{\pi}{\longrightarrow} X)=F_{\operatorname{pr}_{X}}(G \times \mathbb{P} \stackrel{1 \times \pi}{\longrightarrow} G \times X)$. Let $(G \times X) \times_{m} \mathbb{P}$ be defined by the cartesian square

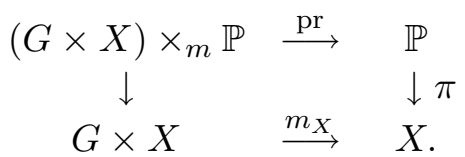

Then the projection $(G \times X) \times_{m} \mathbb{P} \rightarrow G \times X$ represents the functor $F_{m_{X}}$. The corresponding canonical element is $F(\mathrm{pr})(1) \in F\left((G \times X) \times_{m} \mathbb{P} \stackrel{\text { pr }}{\longrightarrow} \mathbb{P} \stackrel{\pi}{\longrightarrow} X\right)=F_{m_{X}}\left((G \times X) \times_{m} \mathbb{P} \rightarrow\right.$ $G \times X)$. Now the functor isomorphism $m$ defines a $(G \times X)$-morphism

$$
G \times \mathbb{P} \rightarrow(G \times X) \times_{m} \mathbb{P}
$$


denoted by $m$ again. The morphism $m$ is determined by the equality $F(m) F(\operatorname{pr})(1)=m(G \times$ $\mathbb{P} \rightarrow G \times X) F\left(\operatorname{pr}_{\mathbb{P}}\right)(1)$. We define $m_{\mathbb{P}}$ to be the composition

$$
G \times \mathbb{P} \stackrel{m}{\longrightarrow}(G \times X) \times_{m} \mathbb{P} \stackrel{\text { pr }}{\longrightarrow} \mathbb{P},
$$

and the assumed associativity property shows that $m_{\mathbb{P}}$ indeed is an action. Thus, claim (a) is proved. For claim (b), let $T \rightarrow X$ be a $G$-scheme over $X$. Then we have

$$
\begin{aligned}
& \operatorname{Mor}_{G, X}(T, \mathbb{P})=\left\{\alpha \in \operatorname{Mor}_{X}(T, \mathbb{P}): \alpha \circ m_{T}=m_{T} \circ(1 \times \alpha)\right\} \\
& \quad=\left\{\alpha \in F(T): F\left(m_{T}\right)(\alpha)=m(G \times T \rightarrow G \times X) F\left(\mathrm{pr}_{T}\right)(\alpha)\right\}
\end{aligned}
$$

(1.5) Let $\mathcal{E}$ be a quasi-coherent $G$-module on $X$. Then the associated projective space bundle $\pi: \mathbb{P}_{X}(\mathcal{E}) \rightarrow X$ represents the functor

$$
\begin{aligned}
F:(\text { Schemes } / X) & \rightarrow \text { Sets } \\
(\rho: T \rightarrow X) & \mapsto\left\{\text { Invertible quotients of } \rho^{*} \mathcal{E}\right\} .
\end{aligned}
$$

The canonical element is the twisting sheaf $\mathcal{O}(1)$ on $\mathbb{P}_{X}(\mathcal{E})$ considered as an invertible quotient of $\pi^{*} \mathcal{E}$. The isomorphism $m_{\mathcal{E}}: m_{X}^{*} \mathcal{E} \stackrel{\sim}{\rightarrow} \operatorname{pr}_{X}^{*} \mathcal{E}$ defines an isomorphism of functors $m: F_{\mathrm{pr}_{X}} \rightarrow$ $F_{m_{X}}$ which satisfies the above associativity property. By Lemma (1.4)(a), we have an action $m_{\mathbb{P}}$ of $G$ on $\mathbb{P}_{X}(\mathcal{E})$ such that the invertible quotient $m_{\mathbb{P}}^{*} \pi^{*} \mathcal{E} \rightarrow m_{\mathbb{P}}^{*} \mathcal{O}(1)$ equals the invertible quotient $m_{\mathbb{P}}^{*} \pi^{*} \mathcal{E}=(1 \times \pi)^{*} m_{X}^{*} \mathcal{E} \stackrel{(1 \times \pi)^{*} m_{\mathcal{E}}}{\longrightarrow}(1 \times \pi)^{*} \operatorname{pr}_{X}^{*} \mathcal{E}=\operatorname{pr}_{\mathbb{P}}^{*} \pi^{*} \mathcal{E} \rightarrow \operatorname{pr}_{\mathbb{P}}^{*} \mathcal{O}(1)$. This means there is an isomorphism $m_{\mathcal{O}(1)}: m_{\mathbb{P}}^{*} \mathcal{O}(1) \stackrel{\sim}{\rightarrow} \operatorname{pr}_{\mathbb{P}}^{*}(\mathcal{O}(1))$ which identifies these two quotient maps. Then $\mathcal{O}(1)$ together with $m_{\mathcal{O}(1)}$ is a $G$-module on $\mathbb{P}_{X}(\mathcal{E})$ and the quotient map $\pi^{*} \mathcal{E} \rightarrow \mathcal{O}(1)$ is a $G$-homomorphism. By Lemma $(1.4)(\mathrm{b})$, the pair $\left(\mathbb{P}_{X}(\mathcal{E}), \pi^{*} \mathcal{E} \rightarrow \mathcal{O}(1)\right)$ together with these $G$-structures represents the functor

$$
\begin{aligned}
(G \text {-schemes } / X) & \rightarrow \text { Sets } \\
(\rho: T \rightarrow X) & \mapsto\left\{\text { Invertible } G \text {-quotients of } \rho^{*} \mathcal{E}\right\}
\end{aligned}
$$

The homomorphism $\mathcal{E} \stackrel{\text { can }}{\longrightarrow} \pi_{*} \pi^{*} \mathcal{E} \longrightarrow \pi_{*} \mathcal{O}(1)$ and, more generally, the homomorphism

$$
\operatorname{Sym}(\mathcal{E}) \rightarrow \Gamma_{*}\left(\mathcal{O}_{\mathbb{P}}\right)=\underset{n \geq 0}{\oplus} \pi_{*}(\mathcal{O}(n))
$$

are $G$-homomorphisms and even $G$-isomorphisms if $\mathcal{E}$ is locally free. Furthermore, it follows that, for any locally free $G$-module $\mathcal{E}$ of rank $r$ on $X$, the Koszul resolution

$$
0 \rightarrow \Lambda^{r} \pi^{*} \mathcal{E} \otimes \mathcal{O}(-r) \rightarrow \ldots \rightarrow \pi^{*} \mathcal{E} \otimes \mathcal{O}(-1) \rightarrow 0
$$

is an exact sequence of $G$-modules on $\mathbb{P}_{X}(\mathcal{E})$.

The same procedure can be applied to other bundle constructions in order to show that they carry a natural $G$-structure and that they satisfy the corresponding universal properties in $(G$-schemes $/ S)$.

(1.6) Let $Y \hookrightarrow X$ be a closed immersion of $G$-schemes over $S$. Using the universal property of blowing up, one easily shows that the blowing up $\mathrm{Bl}_{Y}(X)$ of $X$ along $Y$ carries a natural $G$-action which is compatible with the canonical projection $\mathrm{Bl}_{Y}(X) \rightarrow X$ and that $\mathrm{Bl}_{Y}(X)$ satisfies the obvious universal property in $(G$-schemes $/ S)$.

This fact and the previous considerations show that $G$ naturally acts on the deformation space used for the deformation to the normal cone and that all arrows in the deformation diagram (see page 99 of [FL]) are $G$-morphisms. 


\section{The $\lambda$-Structure on the Equivariant Algebraic $K$-Theory}

After recalling the definition of the higher equivariant $K$-groups, we apply Grayson's techniques of $[\mathrm{Gr}]$ to define exterior power operations on these $K$-groups. We show that these exterior power operations make the equivariant Grothendieck group a $\lambda$-ring and that, on higher $K$-groups, they satisfy two of the three axioms of a $\lambda$-ring. For this, the essential ingredient is the splitting principle based on the equivariant projective space bundle theorem.

As in the previous section, let $S$ be a noetherian scheme and $G$ a flat group scheme over $S$. Let $X$ be a $G$-scheme of finite type over $S$.

(2.1) Definition. For any $q \geq 0$, the $q$-th $K$-group

$$
K_{q}(G, X):=K_{q}(\mathcal{P}(G, X))
$$

(in the sense of Quillen, cf. [Q]) associated with the exact category $\mathcal{P}(G, X)$ consisting of locally free $G$-modules on $X$ of finite rank is called the $q$-th equivariant (algebraic) $K$-group of $X$.

The tensor product makes the Grothendieck group $K_{0}(G, X)$ a commutative ring with $1=\left[\mathcal{O}_{X}\right]$ and $K_{q}(G, X)$ a $K_{0}(G, X)$-module for each $q \geq 0$. We endow the direct sum

$$
K(G, X):=\bigoplus_{q \geq 0} K_{q}(G, X)
$$

with the multiplication induced from this ring and module structures; the product of elements of $\oplus_{q \geq 1} K_{q}(G, X)$ is defined to be zero. The inverse image of $G$-modules obviously makes $K(G,-)$ a contravariant functor with respect to arbitrary $G$-morphisms.

The following theorem is fundamental in the equivariant Riemann-Roch theory.

(2.2) Theorem (Equivariant projective space bundle theorem). Let $\mathcal{E}$ be a locally free $G$ module of rank $d$ on $X$. Let $\pi: \mathbb{P}:=\mathbb{P}_{X}(\mathcal{E}) \rightarrow X$ be the associated projective space bundle in $(G$-schemes $/ S)$ and $\mathcal{O}(1)$ the universal invertible G-module on $\mathbb{P}(\mathrm{cf}$. $(1.5))$. We view $K(G, \mathbb{P})$ as $K(G, X)$-algebra via the pull-back homomorphism $\pi^{*}: K(G, X) \rightarrow K(G, \mathbb{P})$. Then the association $T \mapsto[\mathcal{O}(1)]$ induces an isomorphism

$$
K(G, X)[T] /\left(\sum_{i=0}^{d}(-1)^{i}\left[\Lambda^{i} \mathcal{E}\right] T^{d-i}\right) \stackrel{\sim}{\rightarrow} K(G, \mathbb{P})
$$

of $K(G, X)$-algebras.

Proof. We recall the argument of Thomason (cf. Theorem 3.1 of [Th3]): Quillen's proof of the non-equivariant analogue (cf. [Q], Theorem 2.1) carries over. The essential ingredients Quillen's proof is based on (e. g. the Koszul complex) are summarized in (1.5). Note that even no finiteness assumption on $G$ is necessary though assumed in [Th3].

Next, we recall the notion " $\lambda$-ring".

(2.3) Definition. A $\lambda$-ring is a commutative ring $K$ together with maps

$$
\lambda^{k}: K \rightarrow K, \quad k \geq 0,
$$

which satisfy the following properties:

(i) $\lambda^{0} \equiv 1, \lambda^{1}=\operatorname{id}_{K}, \lambda^{k}(x+y)=\sum_{i=0}^{k} \lambda^{k-i}(x) \cdot \lambda^{i}(y)$ for all $x, y \in K$ and $k \geq 2$.

(ii) $\lambda^{k}(x \cdot y)=P_{k}\left(\lambda^{1} x, \ldots, \lambda^{k} x, \lambda^{1} y, \ldots, \lambda^{k} y\right)$ for all $x, y \in K$ and $k \geq 2$. 
(iii) $\lambda^{k} \lambda^{l}(x)=P_{k, l}\left(\lambda^{1} x, \ldots, \lambda^{k \cdot l} x\right)$ for all $x \in K$ and $k, l \geq 2$.

Here $P_{k}$ and $P_{k, l}$ are the universal integral polynomials defined e. g. on page 5 of [FL]. If $K$ only satisfies axiom (i) we call $K$ a pre- $\lambda$-ring.

(2.4) Lemma. The exterior power operations $[\mathcal{E}] \mapsto\left[\Lambda^{k} \mathcal{E}\right], k \geq 0$, induce well-defined maps $\lambda^{k}, k \geq 0$, on the Grothendieck group $K_{0}(G, X) . K_{0}(G, X)$ together with these maps is a $\lambda$-ring.

Proof. In order to show that $K_{0}(G, X)$ carries a pre- $\lambda$-ring structure, it suffices to show that for any exact sequence $0 \rightarrow \mathcal{E}^{\prime} \rightarrow \mathcal{E} \rightarrow \mathcal{E}^{\prime \prime} \rightarrow 0$ of locally free $G$-modules on $X$ we have

$$
\left[\Lambda^{k} \mathcal{E}\right]=\sum_{i=0}^{k}\left[\Lambda^{k-i} \mathcal{E}^{\prime}\right] \cdot\left[\Lambda^{i} \mathcal{E}^{\prime \prime}\right] \quad \text { in } \quad K_{0}(G, X)
$$

for all $k \geq 0$. In order to prove this (and in order to give at least the essence of Grayson's axioms of power operations on an exact category (cf. [Gr]) needed later on), we will define the obvious equivariant analogue of the Koszul filtration on $\Lambda^{k} \mathcal{E}$ and we will prove the analogous properties: For any $i=0, \ldots, k$, the $G$-module $\mathcal{F}^{i}:=\Lambda^{k-i} \mathcal{E}^{\prime} \wedge \Lambda^{i} \mathcal{E}$ on $X$ is defined to be the image of the canonical $G$-homomorphism $\Lambda^{k-i} \mathcal{E}^{\prime} \otimes \Lambda^{i} \mathcal{E} \rightarrow \Lambda^{k} \mathcal{E}$. Then, similarly to the non-equivariant case, $0 \subseteq \mathcal{F}^{0} \subseteq \ldots \subseteq \mathcal{F}^{k}=\Lambda^{k} \mathcal{E}$ is a filtration of $\Lambda^{k} \mathcal{E}$ by locally free $G$-modules on $X$, and the canonical $G$-homomorphism $\Lambda^{k-i} \mathcal{E}^{\prime} \otimes \Lambda^{i} \mathcal{E} \rightarrow \Lambda^{k-i} \mathcal{E}^{\prime} \otimes \Lambda^{i} \mathcal{E}^{\prime \prime}$ induces a $G$-isomorphism

$$
\mathcal{F}^{i} / \mathcal{F}^{i-1} \stackrel{\sim}{\rightarrow} \Lambda^{k-i} \mathcal{E}^{\prime} \otimes \Lambda^{i} \mathcal{E}^{\prime \prime}
$$

This shows that $K_{0}(G, X)$ is a pre- $\lambda$-ring. Axioms (ii) and (iii) follow from an equivariant version of the splitting principle (see Theorem 2.7 of $[\mathrm{FL}]$ on p. 118) which is a consequence of the equivariant projective space bundle theorem (2.2).

Generalizing the above proof, one easily shows that the category $\mathcal{P}(G, X)$ together with the tensor product $\otimes: \mathcal{P}(G, X) \times \mathcal{P}(G, X) \rightarrow \mathcal{P}(G, X)$ and together with the exterior power operations $\left(\mathcal{E}_{1} \hookrightarrow \ldots \hookrightarrow \mathcal{E}_{k}\right) \mapsto \mathcal{E}_{1} \wedge \ldots \wedge \mathcal{E}_{k}, k \geq 0$, (more or less defined in the proof above) constitutes an exact category with power operations in the sense of Grayson (see section 7 of $[\mathrm{Gr}])$. Associated with these exterior power operations, we have maps

$$
\lambda^{k}: K_{q}(G, X) \rightarrow K_{q}(G, X), \quad k \geq 1, \quad q \geq 0,
$$

constructed by Grayson in section 7 of [Gr]. Similarly to section 8 of [Gr], one easily shows that for $K_{0}(G, X)$ these maps agree with those defined previously. On the higher $K$-groups, these maps are homomorphisms. Thus, we can collect all these maps to define maps

$$
\lambda^{k}: K(G, X) \rightarrow K(G, X), \quad k \geq 1,
$$

such that $K(G, X)$ becomes a pre- $\lambda$-ring. It is easy to see that pull-back homomorphisms are compatible with this $\lambda$-structure.

(2.5) Proposition. The pre- $\lambda$-ring $K(G, X)$ satisfies the axiom (ii) of Definition (2.3).

Proof. Let $x, y \in K(G, X)$. We have to show $\lambda^{k}(x \cdot y)=P_{k}\left(\lambda^{1} x, \ldots, \lambda^{k} x, \lambda^{1} y, \ldots, \lambda^{k} y\right)$. We may assume that $x$ and $y$ are homogeneous. If both $x$ and $y$ lie in $K_{0}(G, X)$, this is already proved in Lemma (2.4). If both $x$ and $y$ are of positive degree, this follows from the fact that the multiplication in positive degrees is defined to be zero. Thus, the only case which remains to be checked is if $x$ of degree 0 and $y$ of degree $q \geq 1$. This is done in section 7 of $[\mathrm{Ko} 5]$ in the non-equivariant case. We recall the essential arguments in order to show that 
this proof carries over to the equivariant case. By the splitting principle, we may assume that $x=[\mathcal{L}]$ where $\mathcal{L}$ is an invertible $G$-module on $X$. Then the formula which has to be shown is $\lambda^{k}(x \cdot y)=x^{k} \cdot \lambda^{k} y$. The well-known isomorphism $\Lambda^{k}(\mathcal{L} \otimes \mathcal{E}) \cong \mathcal{L}^{\otimes k} \otimes \Lambda^{k} \mathcal{E}$ is functorial in $\mathcal{E} \in \mathcal{P}(G, X)$ and $G$-equivariant; therefore, it induces a homotopy on the $K$-theory space which proves this formula.

(2.6) Remark. The above proof heavily relies on the fact that the multiplication on $K(G, X)$ in positive degrees is defined to be trivial. As explained in the last remark of [Ko5], one should be able to prove axiom (ii) already on the simplicial level where such a simplifying fact is not available. Such techniques should also lead to a proof of the following conjecture.

(2.7) Conjecture. The pre- $\lambda$-ring $K(G, X)$ satisfies also the axiom (iii) of Definition (2.3), i. e. $K(G, X)$ is a $\lambda$-ring.

(2.8) Remark.

(a) Let $G$ be a constant group scheme associated with a finite abstract group $\Gamma$ whose order is invertible on $X$. If the $G$-scheme $X$ is affine, one can apply Quillen's construction exposed by Hiller in [Hi] to produce $\lambda$-operations $\lambda^{k}, k \geq 0$, on the higher equivariant $K$-groups $K_{q}(G, X), q \geq 1$. This is explained in detail in section 3 of [Ko2]. Similarly to section 9 of [Gr], one easily shows that these exterior power operations agree with those defined above. Satz (3.5) of [Ko2] shows that Conjecture (2.7) holds in this case. Furthermore, using a version of the Jouanolou construction (cf. section 4 of [Ko2] or $\S 4$ of [We]), one can show that Conjecture (2.7) is true if $X$ is separated and regular.

(b) Let $\mathcal{E}$ be a locally free $G$-module on $X$. The equivariant projective space bundle theorem (2.2) together with Lemma (6.3) and Lemma (6.5) of [AT] show that Conjecture (2.7) is true for $\mathbb{P}_{X}(\mathcal{E})$ if it is true for $X$. More generally, the same holds for Grassmann bundles and flag bundles $D_{\pi}(\mathcal{E})$ of any type $\pi=\left(p_{1}, \ldots, p_{k}\right)$ since the pull-back homomorphism from a flag bundle to the complete flag bundle of $\mathcal{E}$ is injective (see section 3 of [Gro1] and Proposition $(5.8))$.

(c) In Corollary (7.9), we will show that Conjecture (2.7) is true for the $T$-equivariant $K$-theory of generalized flag varieties of the form $G / B$.

(d) Let $S$ be affine or regular and $G$ a diagonalizable group scheme with character group $\Gamma$. Then the canonical decomposition $\mathcal{P}(G, S)=\coprod_{\Gamma} \mathcal{P}(S)$ of categories (see Proposition 4.7.3 in Exp. I of [SGA3] on p. 36) induces an isomorphism $K(G, S) \cong K(S) \otimes \mathbb{Z}[\Gamma]$. Argueing as in the proof of Corollary (7.9), we deduce from this that Conjecture (2.7) is true for $K(G, S)$.

(2.9) Example. Let $S=\operatorname{Spec}(\mathbb{C})$ and let $G$ over $S$ be the constant group scheme associated with a finite group $\Gamma$. Then $K_{0}(G, S)$ is the classical ring of characters $K_{0}(\Gamma, \mathbb{C})$ of the group $\Gamma$. For instance, if $\Gamma$ is cyclic of order $n$, then we have $K_{0}(\Gamma, \mathbb{C})=\mathbb{Z}[T] /\left(T^{n}-1\right)$ where $T$ corresponds to the 1-dimensional representation associated with an injective homomorphism $\Gamma \hookrightarrow \mathbb{C}^{\times}$. In particular, the element $T-1$ is not nilpotent in $K_{0}(\Gamma, \mathbb{C})$. Thus, this example shows that, in contrast to the non-equivariant situation, the elements of the augmentation ideal are in general not nilpotent and that, in particular, the Grothendieck filtration is not locally nilpotent in the equivariant case. 


\section{The Excess Intersection Formula for $G$-Projective Local Com- plete Intersection Morphisms}

First we axiomatically introduce a certain category $\mathcal{C}$ of $G$-schemes which will prove to be an appropriate frame to carry out equivariant Riemann-Roch constructions in it. In particular we show that, for each $X \in \mathcal{C}$, all $G$-(quasi-)projective $G$-schemes over $X$ are again in $\mathcal{C}$. We cite Thomason's paper [Th3] to show that this axiomatic set-up covers many important concrete cases. Then, for any $G$-projective local complete intersection morphism in $\mathcal{C}$, we construct the associated push-forward homomorphism, an equivariant version of the Euler characteristic. Finally, we prove the equivariant excess intersection formula, a rule for commuting pull-back and push-forward homomorphisms.

As in the previous sections, let $S$ be a noetherian scheme and $G$ a flat group scheme over $S$.

(3.1) Notation. Let $\mathcal{C}$ denote the full subcategory of $(G$-schemes $/ S)$ consisting of all $G$ schemes of finite type over $S$ satisfying the following property: Each coherent $G$-module on $X$ is a $G$-quotient of a locally free $G$-module on $X$ (of finite rank).

(3.2) Definition. A $G$-morphism $f: Y \rightarrow X$ of $G$-schemes over $S$ is called $G$-projective (respectively $G$-quasi-projective) if there exists a factorization

$$
Y \stackrel{i}{\hookrightarrow} \mathbb{P}_{X}(\mathcal{E}) \stackrel{\pi}{\rightarrow} X
$$

of $f$ into a closed $G$-immersion $i$ (respectively a locally closed $G$-immersion $i$ ) and the structure morphism $\pi$ of the projective space bundle associated with a locally free $G$-module $\mathcal{E}$ on $X$ (of finite rank).

The following lemma generalizes the usual characterization of (quasi-)projective morphisms to the equivariant case. In Remark (3.5) below, we will quote several hypotheses under which a (quasi-)projective $G$-morphism is $G$-(quasi-)projective.

(3.3) Lemma. Let $X$ be an object in $\mathcal{C}$ and $f: Y \rightarrow X$ a $G$-morphism from a $G$-scheme $Y$ to $X$.

(a) The morphism $f$ is $G$-projective if and only if it is proper and there exists an invertible $G$-module on $Y$ which is very ample relative to $f$.

(b) Let $S$ be separated, $G$ of finite type, separated and faithfully flat over $S$, and $X$ separated over $S$. Then $f$ is $G$-quasi-projective if and only if there exists an invertible $G$-module on Y which is very ample relative to $f$.

Proof. If $f$ is $G$-(quasi-)projective, then $f^{*} \mathcal{O}(1)$ is an invertible $G$-module on $Y$ which is very ample relative to $f$ by definition. This proves one direction in both cases (a) and (b). For the other direction, let $\mathcal{L}$ be a very ample invertible $G$-module on $Y$. Then, by Proposition (4.4.4) of [EGA] II and by (1.5), the adjunction homomorphism $f^{*} f_{*} \mathcal{L} \rightarrow \mathcal{L}$ induces a $G$-immersion $Y \hookrightarrow \mathbb{P}_{X}\left(f_{*} \mathcal{L}\right)$. In case (a), the direct image $f_{*} \mathcal{L}$ is a coherent $G$-module on $X$. Hence, by assumption on $X$, it is a $G$-quotient of a locally free $G$-module $\mathcal{E}$ on $X$. Composing with the induced closed $G$-immersion $\mathbb{P}_{X}\left(f_{*} \mathcal{L}\right) \hookrightarrow \mathbb{P}_{X}(\mathcal{E})$ yields an immersion $Y \hookrightarrow \mathbb{P}_{X}(\mathcal{E})$ which is a closed immersion since $f$ is proper. This proves (a). In case (b), the assumed (finiteness) hypotheses imply that the quasi-coherent $G$-module $f_{*} \mathcal{L}$ is the union of its coherent $G$-submodules (see Lemma 2.1 of [Th3]). Hence, by Proposition (3.8.4) of [EGA] II, there is a coherent $G$-submodule $\mathcal{F}$ of $f_{*} \mathcal{L}$ which, via adjunction, induces a $G$-immersion $Y \hookrightarrow \mathbb{P}_{X}(\mathcal{F})$. Composing with a closed $G$-immersion $\mathbb{P}_{X}(\mathcal{F}) \hookrightarrow \mathbb{P}_{X}(\mathcal{E})$ similarly to case (a) yields the desired immersion in case (b). 
The following lemma assures that the category $\mathcal{C}$ is stable under all equivariant geometric constructions needed for the proof of Riemann-Roch assertions. Furthermore, it shows that $\mathcal{C}$ is rather big if it is not empty.

(3.4) Lemma. Let $X$ be an object in $\mathcal{C}$ and $f: Y \rightarrow X$ a $G$-morphism from a $G$-scheme $Y$ to $X$ over $S$.

(a) If $f$ is $G$-projective, then $Y$ is contained in $\mathcal{C}$. In particular, the blowing up of $X$ along a closed $G$-subscheme is again in $\mathcal{C}$.

(b) Under the hypotheses of Lemma $(3.3)(\mathrm{b})$, we have: If $f$ is $G$-quasi-projective, then $Y$ is contained in $\mathcal{C}$.

Proof. Let $\mathcal{L}$ be a very ample invertible and $\mathcal{F}$ a coherent $G$-module on $Y$. By Propositions (4.6.2) and (4.6.8) of [EGA] II, there is an $n \in \mathbb{N}$ such that the adjunction homomorphism $f^{*} f_{*}\left(\mathcal{F} \otimes \mathcal{L}^{\otimes n}\right) \rightarrow \mathcal{F} \otimes \mathcal{L}^{\otimes n}$ is surjective. In case (a), the direct image $f_{*}\left(\mathcal{F} \otimes \mathcal{L}^{\otimes n}\right)$ is a coherent $G$-module on $Y$. Hence, it is a $G$-quotient of a locally free $G$-module $\mathcal{E}$ on $X$. Thus, $\mathcal{F}$ is a $G$-quotient of the locally free $G$-module $f^{*} \mathcal{E} \otimes \mathcal{L}^{\otimes-n}$. This proves assertion (a). Additional arguments as in the proof of Lemma (3.3)(b) imply assertion (b). For the assertion on blowing up, note that the ideal sheaf of the exceptional divisor is a very ample invertible $G$-module on $X$ (see Proposition (8.1.11) of [EGA] II and (1.6)).

(3.5) Remark. The base scheme $S$ belongs to the category $\mathcal{C}$ in each of the following cases ( $S$ is assumed to be separated in all cases):

(a) $S$ the spectrum of a field $k, G / S$ arbitrary.

(b) $S$ regular of Krull dimension at most one, $G / S$ affine, of finite type and faithfully flat.

(c) $S$ regular or affine, $G / S$ split reductive or isosplit reductive.

(d) $S$ regular or affine, $G / S$ finite and faithfully flat.

(e) $S$ regular or affine, $G / S$ diagonalizable and of finite type.

(f) $S$ regular, $G / S$ of multiplicative type and of finite type.

Here, case (a) is trivial, and the assertions (b) up to (f) are proved in section 5 of [Th3]. Hence, by Lemma (3.4), in all these cases the category $\mathcal{C}$ contains all $G$-quasi-projective $G$-schemes $X$ over $S$ (where, in case (a), we in addition have to assume the hypotheses of Lemma (3.3)(b)). Moreover, if $G$ is smooth, affine and faithfully flat over $S$ with connected fibres, then for each $X \in \mathcal{C}$ all quasi-projective, normal $G$-schemes over $X$ are $G$-quasiprojective over $X$ (by Theorem 1.6 of $[\mathrm{Su}]$ and Lemma (3.3)); thus, they are contained in $\mathcal{C}$ by Lemma (3.4). The same holds (without the assumption "normal") if $G$ is a finite constant group scheme (see the proof of Lemma (1.6) of [Ko2]).

Finally, by Lemma 5.6 of [Th3], in the cases (b) up to (f), the category $\mathcal{C}$ contains all regular separated $G$-schemes of finite type over $S$ (here, in case (b), we in addition have to assume that $G$ is smooth over $S$ ).

Next, for any $G$-projective local complete intersection morphism $f: Y \rightarrow X$ in $\mathcal{C}$, we construct a push-forward homomorphism $f_{*}: K(G, Y) \rightarrow K(G, X)$.

First, let $f=i: Y \hookrightarrow X$ be a regular closed $G$-immersion in $\mathcal{C}$. Let $\mathcal{P}_{\infty}(G, X)$ denote the full subcategory of $\mathcal{M}(G, X)$ consisting of coherent $G$-modules which possess a finite $G$-resolution by locally free $G$-modules on $X$. Then, by Quillen's resolution theorem (cf. Corollary 1, [Q], p. 109), the canonical map $K(G, X) \rightarrow K\left(\mathcal{P}_{\infty}(G, X)\right)$ is an isomorphism. By definition of the category $\mathcal{C}$, for each $\mathcal{F} \in \mathcal{P}(G, Y)$, the direct image $i_{*}(\mathcal{F})$ possesses a $G$-resolution by locally free $G$-modules on $X$; by Schanuel's lemma and the non-equivariant case (see p. 127 of [FL]), it is contained in $\mathcal{P}_{\infty}(G, X)$. Thus, we have an exact functor $i_{*}: \mathcal{P}(G, Y) \rightarrow \mathcal{P}_{\infty}(G, X)$. This functor induces the desired push-forward homomorphism $i_{*}: K(G, Y) \rightarrow K(G, X)$ in this first 
case.

Next, let $f$ be an elementary projection, i. e. let $f=\pi$ be the structure morphism $\mathbb{P}:=$ $\mathbb{P}_{X}(\mathcal{E}) \rightarrow X$ of the projective space bundle associated with a locally free $G$-module $\mathcal{E}$ of rank $d$ on $X$. Let $\mathcal{P}_{0}(G, \mathbb{P})$ be the full subcategory of $\mathcal{P}(G, \mathbb{P})$ consisting of locally free $G$-modules $\mathcal{F}$ with $R^{q} \pi_{*}(\mathcal{F}(k))=0$ for all $q>0$ and $k \geq 0$. Then, by the (generalized) proof of the projective space bundle theorem (see Theorem 2.1 of $[\mathrm{Q}]$, p. 142 ), we have $K(G, \mathbb{P})=K\left(\mathcal{P}_{0}(G, \mathbb{P})\right.$ ), and the direct image functor $\pi_{*}: \mathcal{P}_{0}(G, \mathbb{P}) \rightarrow \mathcal{P}(G, X)$ is well-defined and exact. This functor induces the desired push-forward homomorphism $\pi_{*}: K(G, \mathbb{P}) \rightarrow K(G, X)$ in this second case. For instance, we have $\pi_{*}[\mathcal{O}(n)]=\left[\operatorname{Sym}^{n} \mathcal{E}\right]$ in $K_{0}(G, X)$ for all $n \geq 0$ and $\pi_{*}[\mathcal{O}(n)]=0$ for $n=-1, \ldots,-d+1$ (see $\S 8$ of $[\mathrm{Q}])$.

In the general case, we have a factorization $Y \stackrel{i}{\hookrightarrow} \mathbb{P}_{X}(\mathcal{E}) \stackrel{\pi}{\rightarrow} X$ of $f$ with $i$ of the first and $\pi$ of the second kind. We define $f_{*}:=\pi_{*} \circ i_{*}: K(G, Y) \rightarrow K(G, X)$.

(3.6) Lemma.

(a) The definition of $f_{*}$ does not depend on the chosen factorization of $f$.

(b) $f_{*}$ is functorial for $G$-projective local complete intersection morphisms.

(c) The projection formula holds for $f_{*}$.

Proof. Standard, see Lemma (2.7) of [Ko2].

(3.7) Definition. The push-forward homomorphism $f_{*}: K(G, Y) \rightarrow K(G, X)$ is called the Lefschetz trace of $f$ or the equivariant Euler characteristic.

Finally, to state the excess intersection formula, let

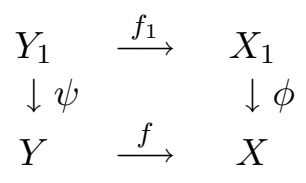

be a cartesian square of $G$-schemes over $S$ with the following properties: All objects $X, Y, X_{1}$, $Y_{1}$ are contained in $\mathcal{C}$, the morphisms $f$ and $f_{1}$ are $G$-projective local complete intersection morphisms, and $\psi$ and $\phi$ are arbitrary $G$-morphisms. We choose a factorization

$$
Y \hookrightarrow \mathbb{P}_{X}(\mathcal{F}) \rightarrow X
$$

of $f$ as above and form the cartesian diagram $\left(\mathcal{F}_{1}:=\phi^{*} \mathcal{F}\right)$

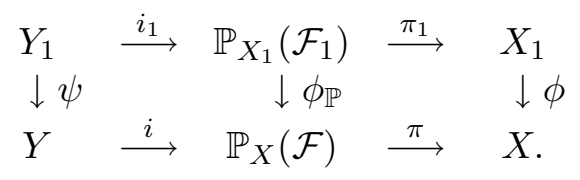

Let $\mathcal{E}$ be the excess conormal sheaf of the left square, i. e.

$$
\mathcal{E}:=\operatorname{ker}\left(\psi^{*} \mathcal{N} \rightarrow \mathcal{N}_{1}\right)
$$

where $\mathcal{N}:=i^{*} \mathcal{I}_{i}$ and $\mathcal{N}_{1}:=i_{1}^{*} \mathcal{I}_{i_{1}}$ are the conormal sheaves of $i$ and $i_{1}$, respectively. Then $\mathcal{E}$ has a natural $G$-structure. As usual (cf. $[\mathrm{FL}]$, p. 153), one shows that the class $[\mathcal{E}] \in K_{0}\left(G, Y_{1}\right)$ does not depend on the chosen factorization of $f$. 
(3.8) Theorem (Equivariant excess intersection formula). The diagram

$$
\begin{array}{crr}
K\left(G, Y_{1}\right) & \stackrel{\left(f_{1}\right)_{*}}{\longrightarrow} & K\left(G, X_{1}\right) \\
\uparrow \lambda_{-1}(\mathcal{E}) \psi^{*} & & \uparrow \phi^{*} \\
K(G, Y) & \stackrel{f_{*}}{\longrightarrow} & K(G, X)
\end{array}
$$

commutes. Here, $\lambda_{-1}(\mathcal{E})$ denotes the element $\sum_{i \geq 0}(-1)^{i}\left[\Lambda^{i} \mathcal{E}\right] \in K_{0}\left(G, Y_{1}\right)$.

Proof. The usual proof (see [FL], chapter VI, §2, and [Ko2], Satz (2.8), and [Ko3], section 2) carries over. To see this, we recall the essential steps: It suffices to prove this formula separately for the left and right square in the above diagram.

The facts needed for the right square are: For any regular (see [Q], p. 138) locally free $G$-module $\mathcal{G}$ on $\mathbb{P}_{X}(\mathcal{F})$, the inverse image $\phi_{\mathbb{P}}^{*}(\mathcal{G})$ is regular again and the base change homomorphism

$$
\phi^{*} \pi_{*}(\mathcal{G}) \rightarrow\left(\pi_{1}\right)_{*} \phi_{\mathbb{P}}^{*}(\mathcal{G})
$$

is a $G$-isomorphism.

The proof of the formula for the left square runs as follows: If the excess dimension is 0 (i. e. if $\mathcal{E}=0$ ), the formula again follows from the fact that the corresponding base change homomorphism is a $G$-isomorphism. If $i$ and $i_{1}$ are elementary embeddings, i. e. if $i$ and $i_{1}$ are zero section embeddings, then $i_{*}(1)$ and $\left(i_{1}\right)_{*}(1)$ can be computed using the Koszul resolution, and the excess intersection formula $\phi_{\mathbb{P}}^{*} i_{*}(y)=\left(i_{1}\right)_{*}\left(\lambda_{-1}(\mathcal{E}) \cdot \psi^{*}(y)\right)$ can be verified for $y=1$ explicitly. Since furthermore, in this situation, $i^{*}$ is surjective the projection formula can be applied to prove this formula for arbitrary $y \in K(G, Y)$. If $i$ and $i_{1}$ are arbitrary regular closed $G$-immersions, the excess intersection formula follows from these two special cases using the so-called deformation to the normal bundle. Note that all constructions needed for this deformation can be carried out equivariantly (by (1.6)) and within the category $\mathcal{C}$ (by Lemma $(3.4))$.

The following corollary is the most important special case of the excess intersection formula.

(3.9) Corollary (Self intersection formula). Let $i: Y \hookrightarrow X$ be a regular closed $G$-immersion in the category $\mathcal{C}$. Let $\mathcal{N}$ be the conormal sheaf of $i$ equipped with the natural $G$-action. Then, for all $y \in K(G, Y)$, we have

$$
i^{*} i_{*}(y)=\lambda_{-1}(\mathcal{N}) \cdot y \quad \text { in } \quad K(G, Y) .
$$

Proof. Apply the theorem to the situation $Y_{1}=X_{1}=Y, f=\phi=i$, and $\psi=f_{1}=\mathrm{id}_{Y}$.

(3.10) Remark. In [Th5], Thomason has shown in the non-equivariant case that the intersection formula already holds on the level of spectra. 


\section{The Equivariant Adams-Riemann-Roch Theorem}

The aim of this section is to formulate and to prove the Adams-Riemann-Roch theorem for $G$-projective local complete intersection morphisms. For this, we first recall the definition of Adams operations and of Bott elements. We show that the $j$-th Bott element of an elementary projection becomes invertible after inverting $j$ and completing the corresponding $K$-group with respect to a certain subideal of the augmentation ideal. Then we are ready to state and to prove the equivariant Adams-Riemann-Roch theorem, a formula for the behaviour of Adams operations with respect to the equivariant Euler characteristic. Finally, we remark that the version given here specializes to former versions of the (equivariant) Adams-Riemann-Roch theorem.

Let $j$ be a natural number and $K$ a pre- $\lambda$-ring. For $x \in K$, we call the expression $\lambda_{t}(x):=$ $\sum_{k \geq 0} \lambda^{k}(x) t^{k} \in K[[t]]$ the $\lambda$-series of $x$. If $\lambda_{t}(x)$ is a polynomial, we call the degree of $\lambda_{t}(x)$ the $\lambda$-degree of $x$.

(4.1) Definition. The $j$-th Adams operation $\psi^{j}$ on $K$ is defined by the recursion formula

$$
\frac{\mathrm{d}}{\mathrm{d} t} \log \lambda_{t}=\sum_{j=1}^{\infty}(-1)^{j-1} \psi^{j} t^{j-1} .
$$

We recall the following properties of $\psi^{j}$ which are certainly well-known if $K$ is even a $\lambda$-ring. Obviously, $\psi^{j}$ is additive, and we have $\psi^{j}(x)=x^{j}$ for all $x \in K$ of $\lambda$-degree $\leq 1$. Let $U_{1}, U_{2}$, ... be indeterminates, and let $N_{j}\left(\lambda^{1}, \ldots, \lambda^{j}\right)=U_{1}^{j}+\ldots+U_{j}^{j}$ be the $j$-th Newton polynomial viewed as a polynomial in the elementary symmetric functions $\lambda^{1}:=U_{1}+\ldots+U_{j}, \ldots, \lambda^{j}:=U_{1}$. $\ldots \cdot U_{j}$. Then the equality $\lambda^{1}+2 \lambda^{2} t+\ldots=\left(1+\lambda^{1} t+\lambda^{2} t^{2}+\ldots\right) \cdot \sum_{j \geq 1}(-1)^{j-1} N_{j}\left(\lambda^{1}, \ldots, \lambda^{j}\right) t^{j-1}$ in $\mathbb{Z}\left[\lambda^{1}, \lambda^{2}, \ldots\right][[t]]$ shows that $\psi^{j}(x)=N_{j}\left(\lambda^{1}(x), \ldots, \lambda^{j}(x)\right)$ for all $x \in K$. If the pre- $\lambda$-ring $K$ in addition satisfies axiom (ii) of Definition (2.3), then a similar argument shows that $\psi^{j}$ is multiplicative. Finally, if $K$ is a $\lambda$-ring, then $\psi^{j}$ is a $\lambda$-ring homomorphism, and we have $\psi^{j} \circ \psi^{k}=\psi^{j k}$ for all $j, k \in \mathbb{N}$ (e. g. see Propositions 5.1 and 5.2 of [AT] on p. 264).

For $d \in \mathbb{N}$, we view the polynomial

$$
\theta^{j, d}\left(\lambda^{1}, \ldots, \lambda^{d}\right):=\prod_{i=1}^{d} \frac{U_{i}^{j}-1}{U_{i}-1}=\prod_{i=1}^{d}\left(1+U_{i}+\ldots+U_{i}^{j-1}\right) \in \mathbb{Z}\left[U_{1}, \ldots, U_{d}\right]
$$

as a polynomial in the elementary symmetric functions $\lambda^{1}, \ldots, \lambda^{d}$ of $\mathbb{Z}\left[U_{1}, \ldots, U_{d}\right]$.

(4.2) Definition. For any $e \in K$ of finite $\lambda$-degree $d$, the element

$$
\theta^{j}(e):=\theta^{j, d}\left(\lambda^{1}(e), \ldots, \lambda^{d}(e)\right) \in K
$$

is called the $j$-th Bott element of $e$.

For example, we have $\theta^{2}(e)=1+\lambda^{1}(e)+\ldots+\lambda^{d}(e) \stackrel{\text { def }}{=} \lambda_{1}(e)$. If $e$ is of $\lambda$-degree 1 , then we have $\theta^{j}(e)=1+e+\ldots+e^{j-1}$, e. g., $\theta^{j}(1)=j$. Obviously, we have $\theta^{j}(e)=\theta^{j, d^{\prime}}(e)$ for all $d^{\prime} \geq d$; therefore, $\theta^{j}$ satisfies the homomorphism property $\theta^{j}\left(e+e^{\prime}\right)=\theta^{j}(e) \cdot \theta^{j}\left(e^{\prime}\right)$ for all $e$, $e^{\prime} \in K$ of finite $\lambda$-degree. If $e=e_{1}-e_{2}$ is the difference of two elements of finite $\lambda$-degree and if $\hat{K}$ is a commutative $K$-algebra such that $\theta^{j}\left(e_{2}\right)$ is invertible in $\hat{K}$, we call

$$
\theta^{j}(e):=\theta^{j}\left(e_{1}\right) \cdot \theta^{j}\left(e_{2}\right)^{-1} \in \hat{K}
$$

the $j$-th Bott element of $e$ in $\hat{K}$. It does not depend on the chosen representation $e=e_{1}-e_{2}$. 
Now, let $K$ be a $\lambda$-ring and $e \in K$ of finite $\lambda$-degree $d$ such that $\lambda^{d}(e)$ is invertible in $K$. Let $I$ denote the ideal of $K\left[j^{-1}\right]$ generated by the elements $\lambda^{1}(e)-\left(\begin{array}{l}d \\ 1\end{array}\right), \ldots, \lambda^{d}(e)-\left(\begin{array}{l}d \\ d\end{array}\right)$.

(4.3) Lemma. Let $L$ be a commutative $K\left[j^{-1}\right]$-algebra such that $I L$ is contained in the Jacobson radical of $L$ (i. e. in the intersection of all maximal ideals of $L$ ). Then the Bott element $\theta^{j}(e)$ is invertible in $L$.

Proof. By the splitting principle (see Theorem 6.1 of $[\mathrm{AT}]$, p. 266), there is a faithfully flat $\lambda$-ring extension $K^{\prime}$ of $K$ such that $e=u_{1}+\ldots+u_{d}$ in $K^{\prime}$ with elements $u_{1}, \ldots, u_{d}$ of $K^{\prime}$ of $\lambda$-degree 1 . Then $\theta^{j}(e)=\prod_{i=1}^{d}\left(1+\left(1+\left(u_{i}-1\right)\right)+\ldots+\left(1+\left(u_{i}-1\right)\right)^{j-1}\right)$ is a symmetric polynomial in $u_{1}-1, \ldots, u_{d}-1$ with constant term $j^{d}$; hence, it is a polynomial in the elementary symmetric functions of $u_{1}-1, \ldots, u_{d}-1$ with constant term $j^{d}$. The $i$-th elementary symmetric function of $u_{1}-1, \ldots, u_{d}-1$ equals the $i$-th Grothendieck operation $\gamma^{i}(e-d)$ (cf. p. 47 of $[\mathrm{FL}])$. Furthermore, one easily verifies the equality

$$
\sum_{i=1}^{d}(-1)^{i} \gamma^{i}(e-d)(t-1)^{d-i}=\sum_{i=0}^{d}(-1)^{i}\left(\lambda^{i}(e)-\left(\begin{array}{l}
d \\
i
\end{array}\right)\right) t^{d-i} \quad \text { in } \quad K[t]
$$

which shows that $\gamma^{i}(e-d)$ is contained in the ideal $I$. Thus, $\theta^{j}(e)$ is invertible in $L \otimes_{K} K^{\prime}$; finally, it is invertible in $L$ because $K^{\prime}$ is faithfully flat over $K$.

Let $\hat{K}\left[j^{-1}\right]$ denote the $I$-adic completion of $K\left[j^{-1}\right]$. Note that first $j$ is inverted and then $K\left[j^{-1}\right]$ is completed though the notation $\hat{K}\left[j^{-1}\right]$ suggests just the other way round. For instance, if the elements $\lambda^{1}(e)-\left(\begin{array}{l}d \\ 1\end{array}\right), \ldots, \lambda^{d}(e)-\left(\begin{array}{l}d \\ d\end{array}\right)$ are nilpotent, then we have $\hat{K}\left[j^{-1}\right]=K\left[j^{-1}\right]$. It is well-known that the $K\left[j^{-1}\right]$-algebra $L=\hat{K}\left[j^{-1}\right]$ satisfies the assumption of Lemma (4.3).

Associated with the element $e$, we have the $\lambda$-ring extension

$$
K[l]:=K[T] /\left(T^{d}-\lambda^{1}(e) T^{d-1}+\ldots+(-1)^{d} \lambda^{d}(e)\right)
$$

of $K$ where the $\lambda$-structure on $K[l]$ is determined by $\lambda_{t}(l)=1+l t$ (see Theorem 2.1 of [FL], p. 8). Then $l$ is invertible in $K[l]$, and the inverse $l^{-1}$ is again of $\lambda$-degree 1 . (Here, the assumption " $\lambda^{d}(e)$ is invertible" is used.)

(4.4) Proposition. The Bott element $\theta^{j}\left(e l^{-1}\right)$ is invertible in the $K[l]$-algebra $\hat{K}[l]\left[j^{-1}\right]:=$ $K[l] \otimes_{K} \hat{K}\left[j^{-1}\right]$.

Proof. The following calculation shows that the element $\left(l^{-1}-1\right)^{d}$ is contained in the ideal $I K[l]\left[j^{-1}\right]$ :

$$
\begin{aligned}
& \left(l^{-1}-1\right)^{d}=(-1)^{d} l^{-d}(l-1)^{d} \\
& \quad=(-1)^{d} l^{-d}\left(l^{d}-d l^{d-1}+\ldots+(-1)^{d}\right) \\
& \quad=(-1)^{d} l^{-d}\left((e-d) l^{d-1}+\ldots+(-1)^{d-1}\left(\lambda^{d}(e)-1\right)\right)
\end{aligned}
$$

The ideal $I \hat{K}\left[j^{-1}\right]$ is contained in the Jacobson radical of $\hat{K}\left[j^{-1}\right]$ which in turn is contained in the Jacobson radical of $\hat{K}[l]\left[j^{-1}\right]$ since $K[l]$ is faithfully flat over $K$. Hence, the $d$-th root $l^{-1}-1$ of $\left(l^{-1}-1\right)^{d}$ is contained in the Jacobson radical of $\hat{K}[l]\left[j^{-1}\right]$. Thus, for each $k \in\{1, \ldots, d\}$, the element

$$
\lambda^{k}\left(e l^{-1}\right)-\left(\begin{array}{l}
d \\
k
\end{array}\right)=\lambda^{k}(e)\left(l^{-k}-1\right)+\left(\lambda^{k}(e)-\left(\begin{array}{l}
d \\
k
\end{array}\right)\right)
$$


is contained in the Jacobson radical of $\hat{K}[l]\left[j^{-1}\right]$. Now Lemma (4.3) proves Proposition (4.4).

Now, let $S$ be a noetherian scheme, $G$ a flat group scheme over $S$, and let $\mathcal{C}$ be the category of $G$-schemes introduced in (3.1). Let $f: Y \rightarrow X$ be a $G$-projective local complete intersection morphism in $\mathcal{C}$. We fix a factorization

$$
Y \stackrel{i}{\hookrightarrow} \mathbb{P}_{X}(\mathcal{E}) \stackrel{\pi}{\rightarrow} X
$$

of $f$ as in Definition (3.2). The formulation of the equivariant Adams-Riemann-Roch theorem (4.5) will depend on this factorization.

Let $\Omega_{\mathbb{P} / X}$ be the module of relative differentials of $\mathbb{P}_{X}(\mathcal{E})$ over $X$ and $\mathcal{N}_{i}:=i^{*}(\mathcal{I}(Y))$ the conormal sheaf of $i$. The element $T_{f}:=\left[i^{*} \Omega_{\mathbb{P} / X}^{\vee}\right]-\left[\mathcal{N}_{i}^{\vee}\right] \in K_{0}(G, Y)$ is called the tangential element of $f$. It does not depend on the chosen factorization of $f$. By the $G$-version of Proposition 3.13 of $[\mathrm{FL}]$, p. 88 , we have $\left[\Omega_{\mathbb{P} / X}\right]=\left[\pi^{*} \mathcal{E} \otimes \mathcal{O}(-1)\right]-1$ in $K_{0}(G, \mathbb{P})$. We put $l:=[\mathcal{O}(1)]$ and $e:=[\mathcal{E}]$ and define $\hat{K}_{0}(G, X)\left[j^{-1}\right]$ to be the completion of $K_{0}(G, X)\left[j^{-1}\right]$ with respect to the ideal $I$ of $K_{0}(G, X)\left[j^{-1}\right]$ generated by the elements $\lambda^{1}(e)-\left(\begin{array}{l}d \\ 1\end{array}\right), \ldots, \lambda^{d}(e)-\left(\begin{array}{l}d \\ d\end{array}\right)$. Analogously, $\hat{K}(G, X)\left[j^{-1}\right]$ is defined. By Proposition (4.4) and the projective space bundle theorem (2.2), the Bott element $\theta^{j}\left(e l^{-1}\right)$ is invertible in $K_{0}(G, \mathbb{P}) \otimes_{K_{0}(G, X)} \hat{K}_{0}(G, X)\left[j^{-1}\right]$. Hence, there is a representation $-T_{f}^{\vee}=e_{1}-e_{2}$ in $K_{0}(G, Y) \otimes_{K_{0}(G, X)} \hat{K}_{0}(G, X)\left[j^{-1}\right]$ with $e_{1}$ and $e_{2}$ of finite $\lambda$-degree and $e_{2}$ invertible. We put

$$
\theta^{j}\left(T_{f}^{\vee}\right)^{-1}:=\theta^{j}\left(-T_{f}^{\vee}\right) \stackrel{\text { def }}{=} j \cdot i^{*}\left(\theta^{j}\left(e l^{-1}\right)\right)^{-1} \cdot \theta^{j}\left(\mathcal{N}_{i}\right) .
$$

The projection formula shows that the push-forward $f_{*}$ defined in section 3 extends to a map

$$
\hat{f}_{*}: K(G, Y) \otimes_{K_{0}(G, X)} \hat{K}_{0}(G, X)\left[j^{-1}\right] \rightarrow \hat{K}(G, X)\left[j^{-1}\right] .
$$

(4.5) Theorem (Equivariant Adams-Riemann-Roch theorem). The diagram

$$
\begin{array}{ccc}
K(G, Y) & \stackrel{\theta^{j}\left(T_{f}^{\vee}\right)^{-1} \cdot \psi^{j}}{\longrightarrow} & K(G, Y) \otimes_{K_{0}(G, X)} \hat{K}_{0}(G, X)\left[j^{-1}\right] \\
\downarrow f_{*} & & \downarrow \hat{f}_{*} \\
& & \hat{K}(G, X)\left[j^{-1}\right]
\end{array}
$$

commutes.

(4.6) Remark.

(a) If $f$ is a regular closed $G$-embedding, we may choose $\mathcal{E}=\mathcal{O}_{X}$. Then $\hat{K}_{0}(G, X)\left[j^{-1}\right]=$ $K_{0}(G, X)\left[j^{-1}\right]$, i. e. Theorem (4.5) holds without completing $K$-theory. Thus, in the case $G$ is a finite constant group scheme whose order is invertible on $S$, Theorem (4.5) specializes to Korollar (5.2) and Satz (5.4)(a) of [Ko2].

(b) If $G$ acts trivially on $X$ and $Y$, then we may choose $\mathcal{E}$ with trivial $G$-action, i. e. the element $e \in K_{0}(G, X)$ comes from $K_{0}(X)$. Then, by Proposition 1.5 of [FL], p. 52, the elements $\lambda^{i}(e)-\left(\begin{array}{c}d \\ i\end{array}\right), i=1, \ldots, d$, are nilpotent and again we have $\hat{K}_{0}(G, X)\left[j^{-1}\right]=K_{0}(G, X)\left[j^{-1}\right]$, i. e. Theorem (4.5) holds without completing $K$-theory. As in (a), here Theorem (4.5) specializes to Satz (5.3) and Satz (5.4)(b) of [Ko2]. If, in particular, $G=S$ is the trivial group scheme, we obtain the usual non-equivariant Adams-Riemann-Roch theorem (see [So], [Ta] and [FL]).

(c) Another special case of Theorem (4.5) is the Adams-Riemann-Roch theorem of Chinburg, 
Erez, Pappas, and Taylor which was independently found. They prove a similar formula for $K_{0}$, if $G$ is a finite constant group scheme, $S$ the spectrum of a Dedekind ring, and $f: X \rightarrow S$ a regular, projective, flat $S$-scheme ([CEPT]).

Proof (of Theorem (4.5)). Since there are some inaccuracies in [FL] in the formulation and in the proof of the Adams-Riemann-Roch theorem for elementary projections, we give the details of this part of the proof.

So first, let $f=\pi$, i. e. $i=\operatorname{id}_{\mathbb{P}_{X}(\mathcal{E})}$. Since the $K(G, X)$-module $K(G, \mathbb{P})$ is generated by the elements $l^{-k}, k=0, \ldots, d-1$, (by the projective space bundle theorem $(2.2)$ ), since $f_{*}$ is $K(G, X)$-linear (by the projection formula), and since $\psi^{j}$ is multiplicative (by Proposition $(2.5))$, it suffices to show

$$
\psi^{j} f_{*}\left(l^{-k}\right)=\hat{f}_{*}\left(j \cdot \theta^{j}\left(e l^{-1}\right)^{-1} \cdot \psi^{j}\left(l^{-k}\right)\right) \quad \text { in } \quad \hat{K}_{0}(G, X)\left[j^{-1}\right]
$$

for all $k=0, \ldots, d-1$. Let $\mathbb{Z}\left[j^{-1}\right]\left[\left[a_{1}, \ldots, a_{d}\right]\right]$ be the formal power series ring in $d$ variables $a_{1}, \ldots, a_{d}$ over $\mathbb{Z}\left[j^{-1}\right]$, and let $s_{1}, \ldots, s_{d}$ be the elementary symmetric functions of $a_{1}, \ldots, a_{d}$. The elements $\gamma^{1}(e-d), \ldots, \gamma^{d}(e-d)$ are contained in the ideal $I$ by the proof of Lemma (4.3). Hence, there is a unique continuous $\mathbb{Z}\left[j^{-1}\right]$-algebra homomorphism

$$
\mathbb{Z}\left[j^{-1}\right]\left[\left[s_{1}, \ldots, s_{d}\right]\right] \rightarrow \hat{K}_{0}(G, X)\left[j^{-1}\right]
$$

which maps $s_{i}$ to $\gamma^{i}(e-d)$ for all $i=1, \ldots, d$. The $K_{0}(G, \mathbb{P})$-algebra

$$
L:=K_{0}(G, \mathbb{P}) \otimes_{K_{0}(G, X)} \hat{K}_{0}(G, X)\left[j^{-1}\right]
$$

is the completion of $K_{0}(G, \mathbb{P})\left[j^{-1}\right]$ with respect to the $I K_{0}(G, \mathbb{P})\left[j^{-1}\right]$-adic topology since $K_{0}(G, \mathbb{P})$ is free over $K_{0}(G, X)$. Since $\left(l^{-1}-1\right)^{d}$ lies in $I K_{0}(G, \mathbb{P})\left[j^{-1}\right]$ (cf. the proof of Proposition (4.4)), the above homomorphism extends to a continuous ring homomorphism

$$
\beta: \mathbb{Z}\left[j^{-1}\right]\left[\left[s_{1}, \ldots, s_{d}, Z\right]\right] \rightarrow L, \quad Z \mapsto l^{-1}-1 .
$$

We define the power series $F_{k}$ to be $(1+Z)^{k j}$ times the inverse of the image of the polynomial $\theta^{j, d}\left(\lambda^{1}, \ldots, \lambda^{d}\right)$ in $\mathbb{Z}\left[j^{-1}\right]\left[\left[s_{1}, \ldots, s_{d}, Z\right]\right]$ under the ring homomorphism

$$
\mathbb{Z}\left[\lambda^{1}, \ldots, \lambda^{d}\right] \rightarrow \mathbb{Z}\left[j^{-1}\right]\left[\left[s_{1}, \ldots, s_{d}, Z\right]\right]
$$

given by

$$
\mathbb{Z}\left[U_{1}, \ldots, U_{d}\right] \rightarrow \mathbb{Z}\left[j^{-1}\right]\left[\left[a_{1}, \ldots, a_{d}, Z\right]\right], \quad U_{i} \mapsto\left(a_{i}+1\right)(Z+1) .
$$

Then we have

$$
\theta^{j}\left(e l^{-1}\right)^{-1} \cdot \psi^{j}\left(l^{-k}\right)=F_{k}\left(\gamma^{1}(e-d), \ldots, \gamma^{d}(e-d), l^{-1}-1\right) \stackrel{\text { def }}{=} \beta\left(F_{k}\right) \quad \text { in } \quad L .
$$

By the division lemma for power series, there are unique elements

$$
b_{0}^{(k)}, \ldots, b_{d-1}^{(k)} \in \mathbb{Z}\left[j^{-1}\right]\left[\left[s_{1}, \ldots, s_{d}\right]\right]
$$

such that $F_{k}$ is congruent to the polynomial $b_{0}^{(k)}+\ldots+b_{d-1}^{(k)} Z^{d-1}$ modulo the symmetric polynomial $\prod_{i=1}^{d}\left((Z+1)\left(a_{i}+1\right)-1\right)$. The image of this symmetric polynomial under $\beta$ is zero by the projective space bundle theorem (2.2). By Lemma 3.3 of [FL] on p. 40, we have

$$
\sum_{\nu=0}^{d-1}(-1)^{\nu} b_{\nu}^{(k)}=\left\{\begin{array}{lll}
j^{-1}, & \text { if } & k=0 \\
0, & \text { if } & k=1, \ldots, d-1
\end{array} .\right.
$$


Hence, we have

$$
\begin{aligned}
\hat{f}_{*}( & \left.\theta^{j}\left(e l^{-1}\right)^{-1} \cdot \psi^{j}\left(l^{-k}\right)\right) \\
& =\hat{f}_{*}\left(\beta\left(b_{0}^{(k)}+\ldots+b_{d-1}^{(k)} Z^{d-1}\right)\right) \\
& =\beta\left(b_{0}^{(k)}\right) \hat{f}_{*}(1)+\ldots+\beta\left(b_{d-1}^{(k)}\right) \hat{f}_{*}\left(\left(l^{-1}-1\right)^{d-1}\right) \\
& =\beta\left(\sum_{\nu=0}^{d-1}(-1)^{\nu} b_{\nu}^{(k)}\right) \\
& =\left\{\begin{array}{lll}
j^{-1}, & \text { if } \quad k=0 \\
0, & \text { if } & k=1, \ldots, d-1 \\
& =j^{-1} \psi^{j} f_{*}\left(l^{-k}\right) .
\end{array}\right.
\end{aligned}
$$

This proves Theorem (4.5) in the case $f=\pi$.

If $f=i$ is a regular embedding, the proof of [FL] carries over to the equivariant case without any major changes (see also [Ko2]). The essential ingredients for this proof are the deformation to the normal cone (see (1.6)) and the excess intersection formula for excess dimension 0 (see (3.8)). Note that only the additivity and multiplicativity of $\psi^{j}$ are used in this proof.

Finally, using the projection formula, these two cases can be put together to prove Theorem (4.5) in the general case.

(4.7) Remark. The given formulation of the equivariant Adams-Riemann-Roch theorem uses the smallest subideal $I$ of the augmentation ideal such that the power series occurring in the proof make sense in the $I$-adic completion of $K$-theory. As explained in Remark (4.6), it specializes to former versions. However, it has the disadvantage that the ideal $I$ depends on the factorization of the morphism $f$ which therefore has to be given. To avoid this, we redefine $\hat{K}_{0}(G, X)\left[j^{-1}\right]$ to be the completion of $K(G, X)\left[j^{-1}\right]$ with respect to the full augmentation ideal in $K_{0}(G, X)$ (see next section). Then, without changes, Theorem (4.5) remains valid. 


\section{The Equivariant Grothendieck-Riemann-Roch Theorem}

First, we show that, under a certain finiteness assumption, the completion of an augmented $\lambda$-ring with respect to the powers of the augmentation ideal is the same as the completion with respect to the Grothendieck filtration. Next, we prove that, after tensoring with $\mathbb{Q}$, the Chern character yields an isomorphism between the completion with respect to the Grothendieck filtration and the completed graded ring associated with the Grothendieck filtration. In particular, the $j^{n}$-eigenspace of the Adams operation $\psi^{j}$ on the completion is independent of $j$. We conjecture that the equivariant Euler characteristic is continuous with respect to the Grothendieck filtrations and we give some examples where this conjecture is true. Assuming this conjecture (and Conjecture (2.7)), we finally state and prove the Grothendieck-Riemann-Roch theorem, a formula for the behaviour of the Chern character with respect to the equivariant Euler characteristic.

By an augmented $\lambda$-ring we mean a $\lambda$-ring $K$ together with a $\lambda$-ring homomorphism $\varepsilon: K \rightarrow$ $\mathbb{Z}$. The associated Grothendieck filtration is denoted by $\left(F^{n}\right)_{n \geq 0}$ (e. g. see [FL], p. 48). In concrete cases, one can usually check that equivariant Grothendieck groups satisfy the finiteness hypothesis assumed in the following proposition.

(5.1) Proposition. Let $(K, \varepsilon)$ be an augmented $\lambda$-ring which is generated as a $\lambda$-ring by elements $y_{i}, i \in I$, of bounded augmentation. Furthermore, we assume that the $\lambda$-degree of $y_{i}$ equals $\varepsilon\left(y_{i}\right)$ for all $i \in I$. Then the canonical map from the $F^{1}$-adic completion of $K$ to the completion of $K$ with respect to the Grothendieck filtration is bijective.

Proof (see also Corollary (12.3) of $[\mathrm{At}]$ ). Let $N:=\max \left\{\varepsilon\left(y_{i}\right): i \in I\right\}$. We will show that

$$
F^{n N} \subseteq\left(F^{1}\right)^{n} \subseteq F^{n}
$$

for all $n \in \mathbb{N}$. Then we are done. The second inclusion is trivial by definition. To prove the first inclusion, we put $x_{i}:=y_{i}-\varepsilon\left(y_{i}\right)$ for $i \in I$. Then we have $K=\mathbb{Z}\left[\lambda^{n}\left(x_{i}\right), i \in I, n \geq 0\right]$. One easily checks that $F^{n N}$ is generated as an abelian group by the monomials

$$
\gamma^{n}(x)^{m}:=\left(\gamma^{n_{1}}\left(x_{i_{1}}\right)\right)^{m_{1}} \cdot \ldots \cdot\left(\gamma^{n_{r}}\left(x_{i_{r}}\right)\right)^{m_{r}}, \quad r, m_{k}, n_{k} \geq 0 \quad \text { with } \quad m_{1} n_{1}+\ldots m_{r} n_{r} \geq n N,
$$

(see also Corollaire 4.15 of [SGA6] on p. 338). We have to show that each of these monomials is contained in $\left(F^{1}\right)^{n}$. If at least one $n_{k}$ is greater than $N$, then $\gamma^{n}(x)^{m}$ vanishes by assumption. Hence, it is certainly contained in $\left(F^{1}\right)^{n}$. If, on the other hand, all $n_{k}$ are less or equal $N$, then we have $m_{1}+\ldots+m_{r} \geq n$ and again we have $\gamma^{n}(x)^{m} \in\left(F^{1}\right)^{n}$. Thus, Proposition (5.1) is proved.

The next lemma presents a general situation when the completion of a filtered ring is isomorphic to the associated completed graded ring.

(5.2) Lemma. Let $K$ be a commutative ring and $\left(F^{n}\right)_{n \geq 0}$ a decreasing filtration of $K$ by ideals $F^{n}, n \geq 0$, with $F^{0}=K$ and $F^{n} \cdot F^{m} \subseteq F^{n+m}$ for all $n, m \geq 0$. Let

$$
\operatorname{ch}=\left(\operatorname{ch}_{n}\right)_{n \geq 0}: K \rightarrow \hat{\operatorname{Gr}} K:=\prod_{n=0}^{\infty} F^{n} / F^{n+1}
$$

be a ring homomorphism with the property $\operatorname{ch}_{n}(x)=x+F^{n+1}$ in $F^{n} / F^{n+1}$ for all $n \geq 0$ and all $x \in F^{n}$. Then ch induces an isomorphism between the completion $\hat{K}$ of $K$ with respect to the $\left(F^{n}\right)_{n \geq 0}$-topology and $\hat{\mathrm{G}} K$. 
Proof. $\hat{\mathrm{Gr}} K$ is separated and complete with respect to the natural topology on $\hat{\mathrm{Gr}} K$. Because of $\operatorname{ch}_{n}\left(F^{k}\right)=0$ for all $n<k$, the ring homomorphism ch extends to a continuous ring homomorphism $\hat{K} \rightarrow \hat{\text { Gr}} K$ denoted by ch again.

To prove injectivity of $\mathrm{ch}$, let $a \in \hat{K}$ with $\operatorname{ch}(a)=0$. We choose a sequence $\left(a_{n}\right)_{n \geq 0}$ in $K$ with $\lim _{n \rightarrow \infty} a_{n}=a$. By passing to a subsequence we may assume that $\operatorname{ch}_{0}\left(a_{n}\right)=0$, $\ldots, \operatorname{ch}_{n-1}\left(a_{n}\right)=0$ for all $n \in \mathbb{N}$. Then, for all $n \in \mathbb{N}$, we have $a_{n} \in F^{n}$ and hence $a=\lim _{n \rightarrow \infty} a_{n}=0$.

The following approximation argument shows the surjectivity of ch. Let $\left(b_{n}\right)_{n \geq 0} \in \hat{\mathrm{Gr}} K$. We choose $a_{0} \in K$ with $a_{0}+F^{1}=b_{0}$ in $F^{0} / F^{1}$. We inductively choose $a_{n} \in F^{n}$ with $a_{n}+F^{n}=b_{n}-\operatorname{ch}_{n}\left(a_{0}+\ldots+a_{n-1}\right)$ in $F^{n} / F^{n+1}$. Then $\left(a_{n}\right)_{n \geq 0}$ is a sequence in $\prod_{n=0}^{\infty} F^{n}$ such that for all pairs $(n, k)$ with $n \leq k$ we have $\operatorname{ch}_{n}\left(a_{0}+\ldots+a_{k}\right)=b_{n}$ in $F^{n} / F^{n+1}$. Thus, the series $\sum_{n=0}^{\infty} a_{n}$ converges in $\hat{K}$ and the limit is a preimage of $\left(b_{n}\right)_{n \geq 0}$ under $\mathrm{ch}$. This ends the proof of Lemma (5.2).

(5.3) Proposition. Let $(K, \varepsilon)$ be an augmented $\lambda$-ring. Then the Chern character induces an isomorphism

$$
\operatorname{ch}: \hat{K}_{\mathbb{Q}} \stackrel{\sim}{\rightarrow} \hat{G r} K_{\mathbb{Q}}
$$

between the completion $\hat{K}_{\mathbb{Q}}$ of $K \otimes \mathbb{Q}$ with respect to the $\left(F_{\mathbb{Q}}^{n}\right)_{n \geq 0}$-topology and the associated completed graded ring $\hat{\operatorname{Gr}} K_{\mathbb{Q}}:=\prod_{n=0}^{\infty} F_{\mathbb{Q}}^{n} / F_{\mathbb{Q}}^{n+1}$.

Proof. By definition, we have $\operatorname{ch}_{n}(x)=\frac{1}{n !} N_{n}\left(c_{1}(x), \ldots, c_{n}(x)\right)$ for all $n \geq 0$ and all $x \in K \otimes \mathbb{Q}$ where $c_{i}(x):=\gamma^{i}(x-\varepsilon(x))+F^{i+1}$ is the $i$-th Chern class of $x$ and $N_{n}$ is the $n$-th Newton polynomial (cf. section 4). For $x \in F^{n} \otimes \mathbb{Q}$, we obviously have $c_{1}(x)=0, \ldots, c_{n-1}(x)=0$ and $c_{n}(x)=(-1)^{n-1}(n-1) ! x+F^{n+1}$ by section 6 of $[\mathrm{Kr}]$. Furthermore, we have $N_{n}\left(0, \ldots, 0, \lambda^{n}\right)=$ $(-1)^{n-1} n \lambda^{n}$. Hence, the Chern character ch satisfies the assumption of Lemma (5.2). Thus, Lemma (5.2) proves Proposition (5.3).

If $K$ is an augmented $\lambda$-ring with locally nilpotent Grothendieck filtration, i. e. if $K$ is the direct limit of $\lambda$-rings with finite Grothendieck filtrations, then Proposition (5.3) shows that the Chern character induces an isomorphism between $K \otimes \mathbb{Q}$ and the associated graded ring $\operatorname{Gr} K_{\mathbb{Q}}:=\oplus_{n \geq 0} F_{\mathbb{Q}}^{n} / F_{\mathbb{Q}}^{n+1}$. Usually, this fact is deduced from the following corollary proved in advance by nilpotent induction (see [FL], [Man] or [Hi]). The argument given here is simpler and generalizes to augmented $\lambda$-rings whose Grothendieck filtration is not locally nilpotent.

\section{(5.4) Corollary.}

(a) For all $n \geq 1$, the eigenspace $\hat{K}_{\mathbb{Q}}^{(n)}$ of the $j$-th Adams operation $\psi^{j}$ on $\hat{K}_{\mathbb{Q}}$ with eigenvalue $j^{n}$ does not depend on $j>1$.

(b) We have $\hat{F}_{\mathbb{Q}}^{m}=\prod_{n \geq m} \hat{K}_{\mathbb{Q}}^{(n)}$ for all $m \geq 0$; in particular, we have $\hat{K}_{\mathbb{Q}}=\prod_{n \geq 0} \hat{K}_{\mathbb{Q}}^{(n)}$; i. e., all Adams operations $\psi^{j}, j \geq 1$, are "diagonalizable" on $\hat{K}_{\mathbb{Q}}$.

Proof. Let $\phi_{n}^{j}: F_{\mathbb{Q}}^{n} / F_{\mathbb{Q}}^{n+1} \rightarrow F_{\mathbb{Q}}^{n} / F_{\mathbb{Q}}^{n+1}$ be the multiplication with $j^{n}$, and let $\phi^{j}:=\prod_{n \geq 0} \phi_{n}^{j}$ : $\hat{\mathrm{Gr}} K_{\mathbb{Q}} \rightarrow \hat{\mathrm{Gr}} K_{\mathbb{Q}}$. Then Corollary (5.4) follows from (5.3) and the commutativity of the diagram

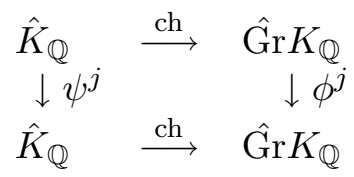

(note that $\psi^{j}$ commutes with natural operations on the category of $\lambda$-rings and that $\psi^{j}$ induces $\phi^{j}$ on $\hat{\mathrm{Gr}} K_{\mathbb{Q}}$ by section 6 of $\left.[\mathrm{Kr}]\right)$. 
(5.5) Example. Let $k$ be a field, and let $\mathbf{G}_{m}$ be the multiplicative group over $k$. Then the Grothendieck group $K_{0}\left(\mathbf{G}_{m}, k\right)$ of representations of $\mathbf{G}_{m}$ on finite dimensional vector spaces over $k$ is isomorphic to the ring of Laurent polynomials $\mathbb{Z}\left[T, T^{-1}\right]$ where $T$ corresponds to the onedimensional representation given by the character id : $\mathbf{G}_{m} \rightarrow \mathbf{G}_{m}$. The augmentation ideal is generated by the element $T-1$ and the completion $\hat{K}_{0}\left(\mathbf{G}_{m}, k\right)$ of $K_{0}\left(\mathbf{G}_{m}, k\right)$ with respect to the Grothendieck filtration is isomorphic to the power series ring $\mathbb{Z}[[U]]$ where $U$ corresponds to $T-1$. The associated completed graded ring is $\mathbb{Z}[[V]]$ where $V$ corresponds to the residue class of $T-1$ modulo $(T-1)^{2}$. The Chern character $\hat{K}_{0}\left(\mathbf{G}_{m}, k\right)_{\mathbb{Q}} \rightarrow \hat{\mathrm{Gr}} K_{0}\left(\mathbf{G}_{m}, k\right)_{\mathbb{Q}}$ is given by $T \mapsto \exp (V)$ or, equivalently, by $U \mapsto \exp (V)-1$. The element

$$
\log (T) \hat{=} \log (1+U):=\sum_{n \geq 1}(-1)^{n-1} \frac{U^{n}}{n}
$$

is a basis of the first Adams eigenspace $\hat{K}_{0}\left(\mathbf{G}_{m}, k\right)_{\mathbb{Q}}^{(1)}$. Finally, the $n$-th power $\log (T)^{n}$ is a basis of the $n$-th Adams eigenspace $\hat{K}_{0}\left(\mathbf{G}_{m}, k\right)_{\mathbb{Q}}^{(n)}$.

Proof. Obvious.

Now, we come back to our standard situation: Let $S$ be a noetherian scheme and $G$ a flat group scheme over $S$. Again, we work in the category $\mathcal{C}$ of $G$-schemes introduced in (3.1). In addition, we assume that all $G$-schemes $X$ considered in this section have the following property: There is a connected component $U$ of $X$ such that the multiplication $G \times U \rightarrow X$ is surjective (set-theoretically). Then, for each $\mathcal{E} \in \mathcal{P}(G, X)$ the rank of $\mathcal{E}$ is a well-defined natural number, and $K_{0}(G, X)$ together with the map rank: $K_{0}(G, X) \rightarrow \mathbb{Z}$ is an augmented $\lambda$-ring. Assuming Conjecture (2.7), also $K(G, X)$ becomes an augmented $\lambda$-ring via the composition $K(G, X) \stackrel{\text { can }}{\longrightarrow} K_{0}(G, X) \stackrel{\text { rank }}{\longrightarrow} \mathbb{Z}$. We denote the $n$-th terms in the associated Grothendieck filtrations by $F_{0}^{n}=F_{0}^{n}(G, X)$ and $F^{n}=F^{n}(G, X)$, respectively. Proposition (5.3) shows that the most natural topology on $K(G, X)$ one should work with is the $\left(F^{n}\right)_{n \geq 0}$-topology. Therefore, as in Remark (4.7), we once more redefine $\hat{K}(G, X)$ to be the completion of $K(G, X)$ with respect to this topology.

Now, let $f: Y \rightarrow X$ be a $G$-projective, local complete intersection morphism in $\mathcal{C}$ of constant relative dimension $d$. As in (4.7), after this redefinition, the equivariant Adams-Riemann-Roch theorem (4.5) for $f$ remains valid without changes, and it does not depend on the chosen factorization of $f$. But it still has the aesthetical disadvantage that its formulation is not functorial: The term in the upper right corner of (4.5) depends not only on $Y$ but also on $X$. This doesn't affect the actual Adams-Riemann-Roch formula since this formula lives in $\hat{K}(G, X)\left[j^{-1}\right]$ anyway. The most natural approach to avoid this disadvantage would be to replace $K(G, Y) \otimes_{K_{0}(G, X)} \hat{K}_{0}(G, X)\left[j^{-1}\right]$ by the completion $\hat{K}(G, Y)\left[j^{-1}\right]$ of $K(G, Y)\left[j^{-1}\right]$ with respect to the $\left(F^{n}(G, Y)\left[j^{-1}\right]\right)_{n \geq 0}$-topology. But then, it is not clear whether, in general, the push-forward homomorphism $f_{*}$ induces a push-forward homomorphism $\hat{f}_{*}$ on the completed objects. Since there are some cases where this can be checked (see below), and since this is a natural hypothesis needed for the equivariant Grothendieck-Riemann-Roch theorem, we state the following conjecture.

(5.6) Conjecture. The equivariant Euler characteristic $f_{*}: K(G, Y) \rightarrow K(G, X)$ is continuous with respect to the Grothendieck filtrations, i. e. for each $n \geq 0$ there is an $m \geq 0$ such that $f_{*}\left(F^{m}(G, Y)\right) \subseteq F^{n}(G, X)$.

(5.7) Remark.

(a) It would also be very useful to get affirmative answers to the following (weaker) questions: 
Is Conjecture (5.6) true for $K_{0}$ ? Is it true after tensoring with $\mathbb{Q}$ ? Is it true for the $F^{1}$-adic topologies? On the other hand, I do not know a counterexample for the following stronger version of (5.6): The $\left(F^{n}\right)_{n \geq 0}$-topology on $K(G, Y)$ is the same as the topology on $K(G, Y)$ induced by pulling back the $\left(F^{n}\right)_{n \geq 0}$-topology on $K(G, X)$.

(b) In the non-equivariant case, the Grothendieck filtration on $K_{0}(Y)$ is nilpotent, if $Y$ has finite dimension (e. g. see Corollary 3.10 of $[\mathrm{FL}]$ on p. 125). Thus, $K_{0}(Y)$ carries the discrete topology and $f_{*}: K_{0}(Y) \rightarrow K_{0}(X)$ certainly is continuous. I do not know, if the latter assertion holds, if the dimension of $Y$ is infinite, or if $K_{0}$ is replaced by higher $K$-theory, though in both cases the Grothendieck filtrations are locally nilpotent and $f_{*}$ even has a degree after tensoring with $\mathbb{Q}$ (see sections 2.1 and 4.4 of [So] and Theorem 3.1 on p. 116 and Theorem 1.1 on p. 135 of $[\mathrm{Ta}])$.

(5.8) Proposition. Conjecture (5.6) is true in the following cases:

(a) The pull-back homomorphism $f^{*}: K(G, X) \rightarrow K(G, Y)$ is surjective. For instance, this holds if $f$ is a regular $G$-embedding which has a $G$-section $p: X \rightarrow Y$ (e. g. if $f$ is a zero section embedding or if $f$ is the embedding of a fixed point).

(b) The morphism $f$ is the structure morphism $D_{\pi}(\mathcal{E}) \rightarrow X$ of the flag bundle $D_{\pi}(\mathcal{E})$ of type $\pi=\left(p_{1}, \ldots, p_{k}\right)$ associated with a locally free $G$-module $\mathcal{E}$ on $X$, see section 3 of [Gro1]. (This includes Grassmann bundles and, in particular, projective space bundles.)

Proof. For (a), note that also $f^{*}: F^{n}(G, X) \rightarrow F^{n}(G, Y)$ is surjective for all $n \geq 0$. Then the projection formula shows that $f_{*}\left(F^{n}(G, Y)\right) \subseteq F^{n}(G, X)$ for all $n \geq 0$. For (b), let $D(\mathcal{E})$ denote the complete flag bundle, and let $g: D(\mathcal{E}) \rightarrow D_{\pi}(\mathcal{E})$ be the canonical projection. Since $D(\mathcal{E})$ considered via $g$ as a $D_{\pi}(\mathcal{E})$-scheme is a product of projective space bundles, we have $f_{*}(y)=f_{*} g_{*}\left(g^{*}(y)\right)$ for all $y \in K\left(G, D_{\pi}(\mathcal{E})\right)$. Now, assertion (b) follows from the projective space bundle theorem $(2.2)$ and Corollary 1.3 of $[\mathrm{FL}]$ on p. 51 since $D(\mathcal{E})$ considered via $f \circ g$ as $X$-scheme can be built up by an iterated construction of projective space bundles.

\section{(5.9) Remark.}

(a) In Proposition (6.4), we will see that Theorem (6.1) of [At] yields a further example where Conjecture (5.6) is true. In Proposition 3.11 of [Ko7], we extend this example from $K_{0}$ - to $K_{1}$-groups.

(b) Chinburg, Erez, Pappas and Taylor have shown ([CEPT]) that Conjecture (5.6) for $K_{0}$ is true, if $S$ is the spectrum of a Dedekind ring $\mathcal{O}, G$ a finite abelian constant group scheme such that the field of fractions of $\mathcal{O}$ is "sufficiently large" with respect to $G$ and if $f: X \rightarrow S$ is a regular, projective, flat $G$-scheme over $S$.

(c) We will prove in section 7 that Conjecture (5.6) is true for $f_{*}: K_{0}(T, G / B) \rightarrow K_{0}(T, S)$; here $G$ is a simply connected split semisimple group scheme over a regular base $S, B$ a Borel subgroup of $G$ and $T$ a maximal split torus in $B$.

(d) In [EG], Edidin and Graham prove a rather general version of Conjecture (5.6) for arbitrary reductive groups acting on smooth varieties over a field.

(5.10) Theorem (Equivariant Grothendieck-Riemann-Roch theorem). We assume that Conjecture (2.7) (for $X$ and $Y$ ) and Conjecture (5.6) are true. Then we have:

(a) The induced push-forward $\left(\hat{f}_{*}\right)_{\mathbb{Q}}: \hat{K}(G, Y)_{\mathbb{Q}} \rightarrow \hat{K}(G, X)_{\mathbb{Q}}$ has degree $d$. Hence, $f_{*}$ induces a graded homomorphism

$$
\hat{\operatorname{Gr}}\left(f_{*}\right)_{\mathbb{Q}}: \hat{\operatorname{Gr}} K(G, Y)_{\mathbb{Q}} \rightarrow \hat{\operatorname{Gr}} K(G, X)_{\mathbb{Q}} .
$$


(b) The diagram

$$
\begin{array}{cc}
K(G, Y) \stackrel{\operatorname{Td}\left(T_{f}\right) \cdot \operatorname{ch}}{\longrightarrow} & \hat{\operatorname{Gr}} K(G, Y)_{\mathbb{Q}} \\
\downarrow f_{*} & \downarrow \hat{\mathrm{Gr}}\left(f_{*}\right)_{\mathbb{Q}} \\
K(G, X) \stackrel{\mathrm{ch}}{\longrightarrow} & \hat{\mathrm{Gr}} K(G, X)_{\mathbb{Q}}
\end{array}
$$

commutes. Here, $\operatorname{Td}\left(T_{f}\right)$ denotes the Todd class of the equivariant tangential element $T_{f} \in$ $K_{0}(G, Y)$ (see section 4 for the definition of $T_{f}$ and p. 20 of [FL] for the definition of $\mathrm{Td}$ ).

Proof. This follows from the equivariant Adams-Riemann-Roch theorem (4.5), Proposition (5.3), and Corollary (5.4) similarly as in Theorem 4.3 of [FL] on p. 65.

(5.11) Example. Let $\operatorname{Pic}(G, X)$ denote the the group of isomorphism classes of invertible $G$-modules on $X$.

If $G$ acts trivially on $X$, then, by Example (1.2)(e), an invertible $G$-module on $X$ is the same as an invertible $\mathcal{O}_{X}$-module together with a homomorphism $G \times X \rightarrow \mathbf{G}_{m, X}$ of group schemes over $X$. For instance: If $G \times X$ is diagonalizable with character group $M$, then we have $\operatorname{Pic}(G, X)=\operatorname{Pic}(X) \times M$. If $G \times X$ is a constant group scheme associated with the abstract group $\Gamma$, then $\operatorname{Pic}(G, X)=\operatorname{Pic}(X) \times \operatorname{Hom}\left(\Gamma^{\mathrm{ab}}, \mathcal{O}_{X}(X)^{\times}\right)$where $\Gamma^{\mathrm{ab}}=\Gamma / \Gamma^{\text {der }}$ denotes the abelianized group.

As usual, one can show that the first Chern class yields an isomorphism between $\operatorname{Pic}(G, X)$ and the first graded piece $F^{1} K_{0}(G, X) / F^{2} K_{0}(G, X)$ of $\hat{\operatorname{Gr}} K_{0}(G, X)$ (see Theorem 1.7 of [FL] on p. 53). The inverse isomorphism is given by the determinant.

Let $\operatorname{Td}\left(T_{f}\right)=1+\tau_{1}+\tau_{2}+\ldots$ be the decomposition of the Todd class into homogeneous components. Then, assuming Conjecture (5.6) (for $K_{0}$ ), the equivariant Grothendieck-RiemannRoch theorem (5.10) yields the formula

$$
c_{1} f_{*}(\mathcal{E})=\hat{\operatorname{Gr}}\left(f_{*}\right)_{\mathbb{Q}}\left(\operatorname{ch}_{d+1}(\mathcal{E})+\operatorname{ch}_{d}(\mathcal{E}) \tau_{1}+\ldots+\tau_{d+1}\right) \quad \text { in } \quad \operatorname{Gr}^{1} K_{0}(G, X)_{\mathbb{Q}}
$$

(for all $\mathcal{E} \in \mathcal{P}(G, Y)$ ) which, via the above isomorphism, can be interpreted as a formula for the determinant of $\hat{f}_{*}[\mathcal{E}]$ in $\operatorname{Pic}(G, X)_{\mathbb{Q}}$. For instance, if $f$ is étale, then we have $\operatorname{Td}\left(T_{f}\right)=1$ and hence

$$
\operatorname{det}_{X}\left(f_{*}(\mathcal{E})\right) \equiv\left[\mathcal{O}_{X}\right]+f_{*}\left(\operatorname{det}_{Y}(\mathcal{E})\right)-f_{*}\left(\mathcal{O}_{Y}\right) \quad \bmod \quad F^{2} K_{0}(G, X)_{\mathbb{Q}}
$$

E. g. we have $\operatorname{det}_{X}\left(f_{*}\left(\mathcal{O}_{\mathcal{Y}}\right)\right) \equiv\left[\mathcal{O}_{X}\right] \bmod F^{2} K_{0}(G, X)_{\mathbb{Q}}$.

Proof. Obvious.

(5.12) Remark (Integral Grothendieck-Riemann-Roch formulas). The Grothendieck-RiemannRoch theorem presented in (5.10) yields a formula in $\hat{\operatorname{Gr}} K(G, X)$ only up to torsion. Therefore, for instance, it usually yields only the non-equivariant information, if $G$ is a finite constant group scheme acting trivially on $X$ (see next section). There are the following approaches to avoid this disadvantage:

(a) In the non-equivariant case, Fulton and MacPherson have given universal integers $L_{n}, n \geq 0$, such that, for all étale morphisms $f: Y \rightarrow X$ and for all locally free modules $\mathcal{E}$ on $Y$, the difference

$$
N_{n}\left(c_{1} f_{*}(\mathcal{E}), \ldots, c_{n} f_{*}(\mathcal{E})\right)-f_{*} N_{n}\left(c_{1}(\mathcal{E}), \ldots, c_{n}(\mathcal{E})\right)
$$

is annihilated by $L_{n}$. Here, $c_{i}$ denotes the $i$-th Chern class in Chow theory (see Theorem 23.3 of $[\mathrm{FM}]$ on p. 76). I hope to be able to prove analogous assertions for equivariant Chern classes in the graded object associated with the Grothendieck filtration. Corollary (6.11) is a first step 
into this direction.

(b) In the situation mentioned in Remark (5.9)(b), Chinburg, Erez, Pappas and Taylor prove a similar Grothendieck-Riemann-Roch formula by tensoring the graded pieces of the graded object with $\mathbb{Z}_{p}\left[\zeta_{p}\right]$ ( $p$ a prime, $\zeta_{p}$ a primitive $p$-th root of unity) in place of $\mathbb{Q}$ ([CEPT]).

(5.13) Remark (Comparison of topological and Grothendieck filtration). Let $X$ be a regular $G$-scheme contained in the category $\mathcal{C}$. By Quillen's resolution theorem (see Corollary 1 on p. 101 of $[\mathrm{Q}])$, we have $K(G, X) \cong K^{\prime}(G, X)$ where $K^{\prime}(G, X)$ denotes the higher $K$-theory associated with the category of coherent $G$-modules on $X$. For any $n \geq 0$, let $F_{\text {top }}^{n} K_{0}(G, X)$ denote the preimage of the subgroup of $K_{0}^{\prime}(G, X)$ generated by coherent sheaves $\mathcal{F}$ whose support has codimension at least $n$ in $X$. Clearly, we have $F_{\text {top }}^{1} K_{0}(G, X) \subseteq F^{1} K_{0}(G, X)$. The reverse inclusion does in general not hold as already seen in Example (2.9).

Let $V$ be a regular connected closed $G$-subscheme of $X$ of codimension $n$. Assuming Conjecture (5.6) to be true for the inclusion $V \hookrightarrow X$, Theorem (5.10)(a) implies that the element $\left[\mathcal{O}_{V}\right]$ of $F_{\text {top }}^{n} K_{0}(G, X)$ is also contained in $F^{n} K_{0}(G, X)_{\mathbb{Q}}$. Note that Conjecture (5.6) is true for instance, if $V$ is a fixed point (see Proposition (5.8)(a)). 


\section{An Induction Formula for (Integral) Representations of Finite Groups}

In this section, we consider a finite group $G$ and explain the meaning of the equivariant AdamsRiemann-Roch theorem (4.5) and the equivariant Grothendieck-Riemann-Roch theorem (5.10) for representations of $G$ on projective modules. Whereas the Grothendieck-Riemann-Roch theorem usually yields only the non-equivariant information, the Adams-Riemann-Roch theorem specializes to an interesting Adams-Riemann-Roch formula for induced representations. In particular, it yields universal annihilators for Chern classes of (integral) induced representations. Furthermore, we investigate the structure of the completed object $\hat{K}_{0}(G, X)\left[j^{-1}\right]$ in various situations. In doing so, we relate some deep results of Atiyah proved in [At] to our considerations in the case $X=\operatorname{Spec}(\mathbb{C})$, and we study the question whether the induction formula mentioned above already holds in classical, i. e. non-completed $K$-theory.

Let $G$ be an (abstract) finite group and $S$ a connected noetherian scheme considered as a $G$-scheme with trivial $G$-action. Then we have natural homomorphisms

$$
i: K(S) \rightarrow K(G, S) \text { and } \varepsilon: K(G, S) \rightarrow K(S)
$$

of augmented (pre)- $\lambda$-rings induced by endowing $\mathcal{O}_{S}$-modules with the trivial $G$-action and by forgetting the $G$-structure, respectively. The induced homomorphisms on the associated graded objects with respect to the $F^{1}$-adic filtrations and with respect to the Grothendieck filtrations will be denoted by $i$ and $\varepsilon$ again. The following proposition generalizes Proposition (6.13) of [At].

(6.1) Proposition. The order of the group $G$ annihilates the kernel of $\varepsilon: \operatorname{Gr} K(G, S) \rightarrow$ $\operatorname{Gr} K(S)$ (with respect to both filtrations).

Proof. Let $\mathcal{O}_{S}[G] \in \mathcal{P}(G, S)$ be the left regular representation of $G$. The maps

$$
\mathcal{O}_{S}[G] \otimes i(\varepsilon(\mathcal{M})) \rightarrow \mathcal{O}_{S}[G] \otimes \mathcal{M}, \quad\left(m_{g}\right)_{g \in G} \mapsto\left(g\left(m_{g}\right)\right)_{g \in G},
$$

for $\mathcal{M} \in \mathcal{P}(G, S)$, define an isomorphism between the exact functors $\mathcal{M} \mapsto \mathcal{O}_{S}[G] \otimes i(\varepsilon(\mathcal{M}))$ and $\mathcal{M} \mapsto \mathcal{O}_{S}[G] \otimes \mathcal{M}$ from $\mathcal{P}(G, S)$ to $\mathcal{P}(G, S)$. Hence, we have $\left[\mathcal{O}_{S}[G]\right] \cdot \alpha=\left[\mathcal{O}_{S}[G]\right] \cdot i(\varepsilon(\alpha))$ in $K(G, S)$ for all $\alpha \in K(G, S)$. Thus, for $\beta \in \operatorname{Gr} K(G, S)$ contained in the kernel of $\varepsilon$ we finally have

$$
\operatorname{ord}(G) \cdot \beta=\left(\operatorname{ord}(G)-\left[\mathcal{O}_{S}[G]\right]\right) \cdot \beta=0
$$

since $\operatorname{ord}(G)-\left[\mathcal{O}_{S}[G]\right]$ is contained in $F_{0}^{1}(G, S)$.

This proposition shows that, after tensoring with $\mathbb{Q}$, the graded object $\operatorname{Gr} K(G, S)$ is isomorphic to the non-equivariant graded object $\operatorname{Gr} K(S)$. In particular, the Grothendieck-Riemann-Roch formula presented in (5.10) yields only the non-equivariant information for any morphism $f$ with target $S$. On the contrary, the Adams-Riemann-Roch theorem (4.5) specializes to an interesting induction formula in representation theory which we explain next.

Let $H$ be a subgroup of $G$, and let $X$ over $S$ be a $G$-scheme of finite type which is contained in the category $\mathcal{C}$ introduced in (3.1) (The latter assumption holds, if $X$ is affine or regular or, more generally, if $X$ has an ample family of invertible $\mathcal{O}_{X}$-modules, see Lemma (2.2) of [Ko2]). Tensoring with $\mathcal{O}_{X}[G]$ over $\mathcal{O}_{X}[H]$ is exact and hence induces a group homomorphism

$$
i_{*}: K(H, X) \rightarrow K(G, X)
$$


which is called the induction map. As in Remark (4.7), for any $j \geq 1$, let $\hat{K}(G, X)\left[j^{-1}\right]$ be the completion of $K(G, X)\left[j^{-1}\right]$ with respect to the $F_{0}^{1}(G, X)\left[j^{-1}\right]$-adic topology.

(6.2) Theorem (Adams-Riemann-Roch formula for induced representations). For all $x \in$ $K(H, X)$ and for all $j \geq 1$, we have

$$
\psi^{j} i_{*}(x)=i_{*} \psi^{j}(x) \quad \text { in } \quad \hat{K}(G, X)\left[j^{-1}\right] .
$$

In particular, we have $\psi^{j}\left(\mathcal{O}_{X}[G / H]\right)=\mathcal{O}_{X}[G / H]$ in $\hat{K}_{0}(G, X)\left[j^{-1}\right]$.

Proof. Let $Y$ be the $G$-scheme $\coprod_{G / H} X=G / H \times X$ where $G$ acts on $G / H$ by left multiplication and on $X$ by the given action. Let $f: Y \rightarrow X$ be the corresponding projection. Then, by Remark (3.5) (see also Remark (6.3) below), the morphism $f$ is a $G$-projective, local complete intersection morphism in $\mathcal{C}$. The $G$-scheme $Y$ is the same as the so-called balanced product $G \times{ }^{H} X$ considered in section 6.1 of [Th3]. By Proposition 6.2 of [Th3], the restriction of $G$ to $H$ and the restriction of $Y$ to the distinguished component $1 H \times X$ in $G / H \times X$ define an isomorphism $r^{*}: K(G, Y) \stackrel{\sim}{\rightarrow} K(H, X)$ of (pre)- $\lambda$-rings such that the diagram

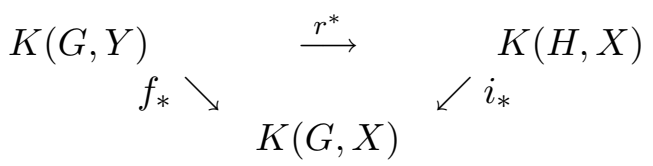

commutes. Now, the equivariant Adams-Riemann-Roch theorem (4.5) applied to the trivial covering $f$ proves Theorem (6.2) since the Bott element of $f$ is 1 .

(6.3) Remark. Let $f$ be the $G$-morphism defined in the proof above. The canonical $G$ isomorphism $\mathcal{O}_{X}[G / H] \stackrel{\sim}{\rightarrow} \prod_{G / H} \mathcal{O}_{X}=f_{*}\left(\mathcal{O}_{Y}\right)$ induces a $G$-surjection $f^{*}\left(\mathcal{O}_{X}[G / H]\right) \rightarrow \mathcal{O}_{Y}$ and hence, by (1.5), a factorization

$$
Y \hookrightarrow \mathbb{P}_{X}\left(\mathcal{O}_{X}[G / H]\right) \rightarrow X
$$

of $f$ as in Definition (3.2). Then, by (4.5), we obtain more precisely that the formula in (6.2) is valid in the completion of $K(G, X)\left[j^{-1}\right]$ with respect to the (smaller) ideal generated by $\left[\Lambda^{i}\left(\mathcal{O}_{X}[G / H]\right)\right]-\left(\begin{array}{c}{[G: H]} \\ i\end{array}\right), i \geq 1$.

For $X=S=\operatorname{Spec}(\mathbb{C})$ the morphism $f$ defined in the proof above yields an example where the push-forward homomorphism $f_{*}$ is continuous as conjectured in (5.6). This is proved in the following proposition using a deep result of Atiyah. However, the associated GrothendieckRiemann-Roch theorem is insignificant because of Proposition (6.1).

(6.4) Proposition. Conjecture (5.6) restricted to $K_{0}$ is true for the $G$-morphism

$$
f: \coprod_{G / H} \operatorname{Spec}(\mathbb{C}) \rightarrow \operatorname{Spec}(\mathbb{C}) .
$$

Proof. As already seen, the push-forward homomorphism

$$
f_{*}: K_{0}\left(G, \coprod_{G / H} \operatorname{Spec}(\mathbb{C})\right) \rightarrow K_{0}(G, \operatorname{Spec}(\mathbb{C}))
$$

can be identified with the induction map $i_{*}: K_{0}(H, \mathbb{C}) \rightarrow K_{0}(G, \mathbb{C})$. By Proposition (5.1), the $\left(F^{n}\right)_{n \geq 0}$-topologies on $K_{0}(H, \mathbb{C})$ and $K_{0}(G, \mathbb{C})$ agree with the $F_{0}^{1}$-adic topologies. Furthermore, by Theorem $(6.1)$ of $[\mathrm{At}]$, the $F_{0}^{1}(H, \mathbb{C})$-adic topology agrees with the $F_{0}^{1}(G, \mathbb{C})$-adic 
topology on $K_{0}(H, \mathbb{C})$ induced by restricting from $G$ to $H$. Now, the projection formula proves Proposition (6.4).

In order to explain the meaning of the induction formula (6.2) in classical representation theory, we next investigate the completion $\hat{K}_{0}(G, k)\left[j^{-1}\right]$ for a field $k$ acted on by $G$ trivially. By [Ke], the isomorphism classes of simple $k G$-modules form a $\mathbb{Z}$-basis of the classical representation ring $K_{0}(G, k)$, and the Adams operations $\psi^{j}, j \geq 0$, on $K_{0}(G, k)$ are periodic. The latter means more precisely: Let $m$ be the char $(k)$-regular exponent of $G$, i. e. the smallest common multiple of the orders of all elements in $G$ whose order is prime to $\operatorname{char}(k)$. Then $\psi^{j+m}=\psi^{j}$ for all $j \geq 0$. In particular, the Adams operation $\psi^{m}$ is the augmentation map composed with the canonical inclusion $\mathbb{Z} \hookrightarrow K_{0}(G, k)$.

(6.5) Lemma. Let $p$ be a prime and $G$ a finite $p$-group. Let $\mathbb{Z}_{p}$ denote the ring of $p$-adic integers. Then we have for all $j \geq 1$ :

$$
\hat{K}_{0}(G, k)\left[j^{-1}\right] \cong \begin{cases}\mathbb{Z}\left[j^{-1}\right] \oplus F_{0}^{1}(G, k) \otimes \mathbb{Z}_{p} & \text { for } p \nmid j j \\ \mathbb{Z}\left[j^{-1}\right] & \text { for } p \mid j .\end{cases}
$$

In particular, the completion map $K_{0}(G, k) \rightarrow \hat{K}_{0}(G, k)\left[j^{-1}\right]$ is injective for $p \nmid j$.

Proof. Because of $K_{0}(G, k)\left[j^{-1}\right]=\mathbb{Z}\left[j^{-1}\right] \oplus F_{0}^{1}(G, k)\left[j^{-1}\right]$, it suffices to show that the $F_{0}^{1}(G, k)$ adic topology on $F_{0}^{1}(G, k)$ is the same as the $p$-adic topology. This is done in Proposition 1.1 of $[\mathrm{AT}]$ on p. 277. (One direction follows from Proposition (6.1), and the other direction follows from the fact that the $\operatorname{ord}(G)$-th power map is the same as the $\operatorname{ord}(G)$-th Adams operation $\bmod p$ which in turn is the same as the augmentation map.)

The induction formula (6.2) says in particular that the induction map $i_{*}$ maps eigenvectors of $\psi^{j}$ to eigenvectors of $\psi^{j}$ with the same eigenvalue. Such eigenvectors are given in the following example based on Lemma (6.5).

(6.6) Example. Let $G$ be a cyclic group of order $p$. Then, for each $j \geq 1$, the completion $\hat{K}_{0}(G, k)\left[j^{-1}\right]$ decomposes into the direct sum of eigenspaces of $\psi^{j}$. More precisely: Let $E_{1}\left(\psi^{j}\right)$ be the eigenspace of $\psi^{j}$ in $\hat{K}_{0}(G, k)\left[j^{-1}\right]$ with eigenvalue 1 , and, for any $(p-1)$-th root of unity $\alpha \neq 1$ in $\mathbb{Z}_{p}$, let $E_{\alpha}\left(\psi^{j}\right)$ be the eigenspace of $\psi^{j}$ in $\hat{F}_{0}^{1}(G, k)\left[j^{-1}\right]=F_{0}^{1}(G, k) \otimes \mathbb{Z}_{p}$ with eigenvalue $\alpha$. Then we have:

$$
\hat{K}_{0}(G, k)\left[j^{-1}\right]=\oplus_{\alpha \in \mu_{p-1}\left(\mathbb{Z}_{p}\right)} E_{\alpha}\left(\psi^{j}\right) .
$$

Proof. The case $p \mid j$ being trivial by Lemma (6.5), we may assume that $p \nmid j$. Then we have $\left(\psi^{j}\right)^{p-1}=\psi^{\left(j^{p-1}\right)}=\psi^{1}=$ id since the Adams operations are periodic of length $p$. Hence, the polynomial $T^{p-1}-1$ annihilates $\psi^{j}$. But $T^{p-1}-1$ equals $\prod_{\alpha \in \mu_{p-1}\left(\mathbb{Z}_{p}\right)}(T-\alpha)$ in $\mathbb{Z}_{p}[T]$, and the ideals $(T-\alpha), \alpha \in \mu_{p-1}\left(\mathbb{Z}_{p}\right)$, in $\mathbb{Z}_{p}[T]$ are pairwise comaximal. Now, the Chinese remainder theorem proves Example (6.6).

In the classical case $k=\mathbb{C}$, we may identify representations of the group $G$ with their associated characters. Then the kernel of the completion map can be described in the following way.

(6.7) Lemma. For any $j \geq 1$, we have:

$$
\begin{aligned}
& \operatorname{ker}\left(K_{0}(G, \mathbb{C}) \rightarrow \hat{K}_{0}(G, \mathbb{C})\left[j^{-1}\right]\right)=\left\{\chi \in K_{0}(G, \mathbb{C}): \chi(g)=0\right. \\
& \quad \text { for all } g \in G \text { whose order is a power of a prime not dividing } j\} .
\end{aligned}
$$

Proof. By Proposition (6.10) of [At], the intersection of all powers of the augmentation ideal $I$ is given by

$$
\cap_{n=1}^{\infty} I^{n}=\left\{\chi \in K_{0}(G, \mathbb{C}): \chi(g)=0 \text { for all } g \in G \text { whose order is a power of a prime }\right\} .
$$


This proves Lemma (6.7) for $j=1$. An easy generalization of Atiyah's arguments proves Lemma (6.7) for arbitrary $j$.

Next, we investigate the question whether the induction formula (6.2) (for $K_{0}$ ) already holds in $K_{0}(G, X)$ (without inverting $j$ and completing).

(6.8) Example. Let $\chi$ be the character of a complex representation of the subgroup $H$ of $G$. Then the character of the induced representation is given by

$$
G \rightarrow \mathbb{C}, \quad g \mapsto \frac{1}{\operatorname{ord}(H)} \sum_{x \in G: x^{-1} g x \in H} \chi\left(x^{-1} g x\right)
$$

(e. g. see formula (10.3) of [CR] on p. 230). The character of $\psi^{j}(\chi)$ is given by

$$
H \rightarrow \mathbb{C}, \quad h \mapsto \chi\left(h^{j}\right)
$$

(e. g. Proposition (12.8) of [CR]). If $j$ is coprime to the order of $G$, then the condition $x^{-1} g x \in H$ is equivalent to the condition $x^{-1} g^{j} x \in H$. Hence, in this case, the induction formula (6.2) applied to the situation $X=S=\operatorname{Spec}(\mathbb{C})$ already holds in $K_{0}(G, \mathbb{C})$. On the other hand, one easily sees that, in general, it is not true with $\hat{K}_{0}(G, \mathbb{C})\left[j^{-1}\right]$ replaced by $K_{0}(G, \mathbb{C})$. Lemma (6.7) shows that the error term is killed by the completion map $K_{0}(G, \mathbb{C}) \rightarrow \hat{K}_{0}(G, \mathbb{C})\left[j^{-1}\right]$. Thus, in this classical situation, we have proved the induction formula (6.2) without using the Riemann-Roch formalism developed in the previous sections. The same applies to $K_{1}$-groups by Theorem 3.10 in [Ko7].

In the following example, we rather explicitly determine the structure of $K_{0}(G, \mathbb{Z})$ and describe the Adams operations on it, if $G$ is a cyclic group of order $p$. Thereby, we show that the induction formula $\psi^{j}(\mathbb{Z}[G])=\mathbb{Z}[G]$ is already valid in $K_{0}(G, \mathbb{Z})$, if $p \nmid j$.

(6.9) Example. Let $p$ be a prime and $G$ a cyclic group of order $p$. Let $\varepsilon$ denote the canonical $\lambda$-ring homomorphism $K_{0}(G, \mathbb{Z}) \rightarrow K_{0}(G, \mathbb{Q})$.

(a) We have a ring isomorphism $K_{0}(G, \mathbb{Q}) \cong \mathbb{Z}[X] /((X+1)(X-p+1))$ where $X$ corresponds to the representation $\mathbb{Q}\left(\zeta_{p}\right)$ of $G$ ( $\zeta_{p}$ a primitive $p$-th root of unity). The $j$-th Adams operation $\psi^{j}$ on $K_{0}(G, \mathbb{Q})$ is the identity, if $p \nmid j$, and it is given by $X \mapsto p-1$, if $p \mid j$.

(b) There is a $\lambda$-ring homomorphism $i: K_{0}(G, \mathbb{Q}) \rightarrow K_{0}(G, \mathbb{Z})$ which splits $\varepsilon$. In particular, $\varepsilon$ is surjective.

(c) We have $\psi^{j}(\mathbb{Z}[G])=\mathbb{Z}[G]$ in $K_{0}(G, \mathbb{Z})$, if $p \nmid j$.

(d) The kernel $C$ of $\varepsilon$ is naturally isomorphic to the classgroup of the $p$-th cyclotomic field $\mathbb{Q}\left(\zeta_{p}\right)$. If $p \nmid j$, the induced $j$-th Adams operation $\psi^{j}$ on $C$ is the composition of the multiplication with $j$ and the automorphism induced by the Galois automorphism $\sigma_{j^{-1}}$ of $\mathbb{Q}\left(\zeta_{p}\right)$ corresponding to $\bar{j}^{-1}$ via the canonical isomorphism $\operatorname{Gal}\left(\mathbb{Q}\left(\zeta_{p}\right) / \mathbb{Q}\right) \cong(\mathbb{Z} / p \mathbb{Z})^{\times}$. If $p \mid j$, the $j$-th Adams operation $\psi^{j}$ on $C$ is the trivial homomorphism.

\section{Proof.}

(a) This is well-known and easy to prove.

(b) If, more generally, $G$ is an abelian group, the permutation modules $\mathbb{Q}[G / H], H$ a subgroup of $G$ with $G / H$ cyclic, form a basis of $K_{0}(G, \mathbb{Q})$. Thus, we can define a splitting $i$ of $\varepsilon$ by $i(\mathbb{Q}[G / H]):=\mathbb{Z}[G / H]$. Then we obviously have $i(\mathbb{Q}[G / H])=\mathbb{Z}[G / H]$ for all subgroups $H$ of $G$. We will show in the proof of assertion (d) that $i$ is a $\lambda$-ring homomorphism, if $G$ is cyclic of order $p$.

(c) The formula $\psi^{j}(\mathbb{Q}[G])=\mathbb{Q}[G]$ is certainly valid in $K_{0}(G, \mathbb{Q})$ for $p \nmid j$ by assertion (a). (Alternatively, this follows from Example (6.8) for an arbitrary group $G$ since the canonical 
$\lambda$-ring homomorphism $K_{0}(G, \mathbb{Q}) \rightarrow K_{0}(G, \mathbb{C})$ is injective by Théorème I of $[\mathrm{Ke}]$ on p. 5.) Now, (the proof of) assertion (b) proves assertion (c).

(d) Since $\mathbb{Z}$ is regular, we have $K_{0}(G, \mathbb{Z})=G_{0}(\mathbb{Z}[G])$ by Satz (2.1) of [Ko2]. By Lenstra's formula (see $[$ Le $]$ or $[$ Ko 4$]$ ), we have $G_{0}(\mathbb{Z}[G]) \cong G_{0}(\mathbb{Z}) \oplus G_{0}\left(\mathbb{Z}\left[p^{-1}\right]\left[\zeta_{p}\right]\right)$. Since $\mathbb{Z}\left[p^{-1}\right]\left[\zeta_{p}\right]$ is a Dedekind ring, we have $G_{0}\left(\mathbb{Z}\left[p^{-1}\right]\left[\zeta_{p}\right]\right) \cong K_{0}\left(\mathbb{Z}\left[p^{-1}\right]\left[\zeta_{p}\right]\right) \cong \mathbb{Z} \oplus \operatorname{Pic}\left(\mathbb{Z}\left[p^{-1}\right]\left[\zeta_{p}\right]\right)$ (see Proposition (2.1) of $[\mathrm{Ba}]$ on p. 453 and Corollary (3.8) of $[\mathrm{Ba}]$ on p. 468). The Picard group $\operatorname{Pic}\left(\mathbb{Z}\left[p^{-1}\right]\left[\zeta_{p}\right]\right)$ is the same as the class group of $\mathbb{Z}\left[\zeta_{p}\right]$ since the only prime ideal of $\mathbb{Z}\left[\zeta_{p}\right]$ lying over $p$ is a principal ideal. This together with assertion (a) proves the first assertion in (d).

In order to show the remaining assertions of (d) (and (b)), we split $\mathbb{Z}[G]$-modules into eigenspaces by tensoring them with $\mathbb{Z}\left[p^{-1}\right]\left[\xi_{p}\right]$ over $\mathbb{Z}$ where $\xi_{p}$ is another primitive $p$-th root of unity. For this, we consider the following commutative diagram of $K$-groups:

$$
\begin{aligned}
& 0 \rightarrow \quad \operatorname{Pic}\left(\mathbb{Z}\left[\zeta_{p}\right]\right) \quad \stackrel{\alpha}{\rightarrow} \quad K_{0}(G, \mathbb{Z}) \quad \stackrel{\varepsilon}{\rightarrow} \quad K_{0}(G, \mathbb{Q}) \quad \rightarrow 0 \\
& \downarrow \otimes \mathbb{Z}\left[p^{-1}\right]\left[\xi_{p}\right] \quad \downarrow \otimes \mathbb{Q}\left(\xi_{p}\right) \\
& \downarrow \beta \quad K_{0}\left(G, \mathbb{Z}\left[p^{-1}\right]\left[\xi_{p}\right]\right) \quad \rightarrow \quad K_{0}\left(G, \mathbb{Q}\left(\xi_{p}\right)\right) \\
& 0 \rightarrow \prod_{a \in \mathbb{Z} / p \mathbb{Z}} \operatorname{Pic}\left(\mathbb{Z}\left[\xi_{p}\right]\right) \stackrel{\gamma}{\rightarrow} \quad \prod_{a \in \mathbb{Z} / p \mathbb{Z}} K_{0}\left(\mathbb{Z}\left[p^{-1}\right]\left[\xi_{p}\right]\right) \quad \rightarrow \quad \prod_{a \in \mathbb{Z} / p \mathbb{Z}} K_{0}\left(\mathbb{Q}\left(\xi_{p}\right)\right) \rightarrow 0
\end{aligned}
$$

Here, the horizontal exact sequences come from the above considerations. Recall that the homomorphism $\alpha: \operatorname{Pic}\left(\mathbb{Z}\left[\zeta_{p}\right]\right) \rightarrow K_{0}(G, \mathbb{Z})$ is given by $M \mapsto[M]-\left[\mathbb{Z}\left[\zeta_{p}\right]\right]$ where the modules $M$ and $\mathbb{Z}\left[\zeta_{p}\right]$ are considered as $\mathbb{Z}[G]$-modules via the ring homomorphism $\mathbb{Z}[G] \rightarrow \mathbb{Z}\left[\zeta_{p}\right], g \mapsto \zeta_{p}$, ( $g$ a fixed generator of $G$ ). The map $\gamma$ is the product of analogously defined homomorphisms. The vertical equalities are given by multiplying a $G$-module $M$ with the idempotents $e_{a}:=p^{-1} \sum_{i=0}^{p-1} \xi_{p}^{-i a} g^{i}, a \in \mathbb{Z} / p \mathbb{Z}$. The map $\beta$ is defined as follows: Let $M$ be an invertible $\mathbb{Z}\left[\zeta_{p}\right]$-module. For $a \in(\mathbb{Z} / p \mathbb{Z})^{\times}$the $\mathbb{Z}\left[\xi_{p}\right]$-module $M_{a}$ is defined to be the abelian group $M$ together with the $\mathbb{Z}\left[\xi_{p}\right]$-structure induced by the ring homomorphism $\mathbb{Z}\left[\xi_{p}\right] \rightarrow \mathbb{Z}\left[\zeta_{p}\right], \xi_{p} \mapsto \zeta_{p}^{\left(a^{-1}\right)}$. For $a=0 \in \mathbb{Z} / p \mathbb{Z}$ the $\mathbb{Z}\left[\xi_{p}\right]$-module $M_{a}$ is defined to be the trivial module $\mathbb{Z}\left[\xi_{p}\right]$. Then the

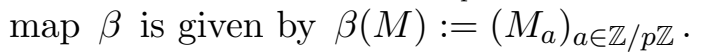

The commutativity of the left square follows from the $\mathbb{Z}\left[p^{-1}\right]\left[\xi_{p}\right]$-isomorphisms

$$
M_{a}\left[p^{-1}\right] \stackrel{\sim}{\rightarrow} e_{a}\left(\mathbb{Z}\left[p^{-1}\right]\left[\xi_{p}\right] \otimes M\right), \quad m \mapsto e_{a}(1 \otimes m)
$$

(for $\left.a \in(\mathbb{Z} / p \mathbb{Z})^{\times}\right)$and the independence of $\mathbb{Z}\left[\xi_{p}\right]_{a}$ of $a \in \mathbb{Z} / p \mathbb{Z}$. The commutativity of the right squares is clear.

Now, the obvious but crucial fact is that all vertical arrows are injective. Therefore, in order to show that the splitting $i$ defined above is a $\lambda$-ring homomorphism, it suffices to note that the obvious inclusion $K_{0}\left(G, \mathbb{Q}\left(\xi_{p}\right)\right)=\mathbb{Z}\left[\operatorname{Hom}\left(G, \mathbb{Q}\left(\xi_{p}\right)^{\times}\right)\right]=\mathbb{Z}\left[\operatorname{Hom}\left(G, \mathbb{Z}\left[p^{-1}\right]\left[\xi_{p}\right]^{\times}\right)\right] \hookrightarrow$ $K_{0}\left(G, \mathbb{Z}\left[p^{-1}\right]\left[\xi_{p}\right]\right)$ is a $\lambda$-ring homomorphism which extends the splitting $i$. In order to verify the claimed action of the Adams operation $\psi^{j}$ on $\operatorname{Pic}\left(\mathbb{Z}\left[\zeta_{p}\right]\right)$, it suffices to note that $\psi^{j}\left(\gamma\left(\left(M_{a}\right)_{a \in \mathbb{Z} / p \mathbb{Z}}\right)\right)$ equals $\gamma\left(\left(M_{j^{-1} a}^{\otimes j}\right)_{a \in \mathbb{Z} / p \mathbb{Z}}\right)$, if $p \nmid j$, and that it equals

$$
\left.\gamma\left(\left(\otimes_{a \in \mathbb{Z} / p \mathbb{Z}} M_{a}^{\otimes j}\right), \mathbb{Z}\left[\xi_{p}\right], \ldots, \mathbb{Z}\left[\xi_{p}\right]\right)\right)=\gamma\left(\left(\mathbb{Z}\left[\xi_{p}\right], \ldots, \mathbb{Z}\left[\xi_{p}\right]\right)\right)=0,
$$

if $p \mid j$. 
(6.10) Remark. In forthcoming papers, we will prove the following (stronger) versions of the induction formula (6.2):

(a) The equality $\psi^{j}\left(\mathcal{O}_{X}[G / H]\right)=\mathcal{O}_{X}[G / H]$ holds already in $K_{0}(G, X)$ if $H$ is a normal subgroup and $\operatorname{gcd}(j, \operatorname{ord}(G / H))=1$ (see Remark 1.8 in [Ko9]).

(b) Let $j$ be a prime which does not divide ord $(G)$ and let $C$ be a cyclic group of order $j$. Then, for all $x \in K_{0}(H, X)$, we have $\psi^{j} i_{*}(x)=i_{*} \psi^{j}(x)$ in $K_{0}(C \times G, X) /\left(\mathcal{O}_{X}[C]\right)$ (see [Ko10]).

The essential ingredient in the proof of (6.2) being the triviality of the Bott element of the trivial covering $f$, strengthened versions of Theorem (6.2) might even exist for étale equivariant morphisms $f$. The techniques of $[\mathrm{FM}]$ mentioned also in Remark (5.12)(a) could help to find general conditions which would imply the vanishing of the difference $\psi^{j} f_{*}-f_{*} \psi^{j}$ without completing $K$-theory. In Theorem 2.33 of [Sn], Snaith shows that "the Brauer induction theorem commutes with Adams operations". A generalization of the canonical induction procedure used for this theorem (see also [Bo], especially section 4.3) could lead to strengthened versions of (6.2), too.

In the remainder of this section, we will apply the Adams-Riemann-Roch formula (6.2) for $K_{0}$ to get universal annihilators for Chern classes of induced representations.

(6.11) Corollary. For any $k \geq 1$, let $M_{k}$ be the natural number

$$
M_{k}:= \begin{cases}2 \cdot \prod_{p \text { prime: }(p-1) \mid k} p^{\operatorname{ord}_{p}(k)+1,}, & \text { if } k \text { is even } \\ 2, & \text { if } k \text { is odd }\end{cases}
$$

Then, for all $G, H, X$ as in Theorem (6.2), we have

$$
M_{k} \cdot N_{k}\left(c_{1}\left(\mathcal{O}_{X}[G / H]\right), \ldots, c_{k}\left(\mathcal{O}_{X}[G / H]\right)\right)=0 \quad \text { in } \quad \operatorname{Gr}^{k} K_{0}(G, X)=F_{0}^{k}(G, X) / F_{0}^{k+1}(G, X)
$$

Proof. Since the group homomorphism $s_{k}:=N_{k}\left(c_{1}, \ldots, c_{k}\right)$ obviously annihilates $F_{0}^{k+1}(G, X)$, it induces a homomorphism

$$
s_{k}: \hat{K}_{0}(G, X)\left[j^{-1}\right] \rightarrow \mathrm{Gr}^{k} K_{0}(G, X) \otimes \mathbb{Z}\left[j^{-1}\right]
$$

for all $j \geq 1$. By Theorem (6.2) and Proposition 6.3 of [FL] on p. 25, we have

$$
s_{k}\left(\mathcal{O}_{X}[G / H]\right)=s_{k}\left(\psi^{j}\left(\mathcal{O}_{X}[G / H]\right)\right)=j^{k} s_{k}\left(\mathcal{O}_{X}[G / H]\right) \quad \text { in } \quad \operatorname{Gr}^{k} K_{0}(G, X) \otimes \mathbb{Z}\left[j^{-1}\right]
$$

for all $j \geq 1$. Hence, for each $j \geq 1$ there is a $n_{j} \in \mathbb{N}$ such that

$$
j^{n_{j}}\left(j^{k}-1\right) s_{k}\left(\mathcal{O}_{X}[G / H]\right)=0 \text { in } \operatorname{Gr}^{k} K_{0}(G, X) .
$$

By section 3.3 of [Th1], we have $\operatorname{gcd}\left\{j^{\infty}\left(j^{k}-1\right): j=1,2, \ldots\right\}=M_{k}$. Thus, Corollary (6.11) is proved.

The annihilators of the elements $s_{k}\left(\mathcal{O}_{X}[G / H]\right), k \geq 1$, presented in the previous corollary induce universal annihilators of the Chern classes $c_{k}\left(\mathcal{O}_{X}[G / H]\right), k \geq 1$, as follows.

(6.12) Corollary. For any $k \geq 1$, let $L_{k}$ be the natural number

$$
L_{k}:=k \cdot \operatorname{lcm}\left\{M_{l}: 1 \leq l \leq k\right\} .
$$

Then, for all $G, H, X$ as in Theorem (6.2) we have

$$
L_{k} \cdot c_{k}\left(\mathcal{O}_{X}[G / H]\right)=0 \text { in } \operatorname{Gr}^{k} K_{0}(G, X) .
$$


Proof. This immediately follows form Corollary (6.11) by Newton's recursion formula

$$
N_{k}\left(X_{1}, \ldots, X_{k}\right)-N_{k-1}\left(X_{1}, \ldots, X_{k-1}\right) X_{1}+\ldots+(-1)^{k-1} N_{1}\left(X_{1}\right) X_{k-1}+(-1)^{k} k X_{k}=0 .
$$

\section{(6.13) Remark.}

(a) For $k$ even, the universal annihilator $M_{k}$ equals the denominator of $B_{k} / 2 k$ where $B_{k}$ is the $k$-th Bernoulli number (see section 3.3 of [Th1]).

(b) If there exists an Adams-Riemann-Roch formula for induced representations without denominators as suggested by Example (6.8), Example (6.9)(c) and Remark (6.10), then the proof of Corollary (6.11) could be applied not only to the homomorphisms $s_{k}=N_{k}\left(c_{1}, \ldots, c_{k}\right), k \geq 1$, but already to the Chern classes $c_{k}, k \geq 1$, and we would obtain improved annihilators for the Chern classes $c_{k}\left(\mathcal{O}_{X}[G / H]\right), k \geq 1$.

(c) The above corollaries are not only valid for the graded object associated with the Grothendieck filtration on $K_{0}(G, X)$ but for all equivariant cohomology theories reasonable equivariant Chern classes can be defined within. For instance, for any prime $l$ which is invertible on $X$ we have the equivariant $l$-adic Chern classes in $H^{*}\left(X_{\text {ét }}, G, \mathbb{Z}_{l}(-)\right)$ due to Grothendieck (see $\S 2$ and $\S 3$ of [Gro2]) or, if $X$ is a $\mathbb{C}$-scheme, we have the transcendental Chern classes in $H^{*}\left(X^{\text {an }}, G, \mathbb{Z}\right)$ (see $\S 3$ of [Gro2] or the appendix of $[\mathrm{At}]$, if $X=\operatorname{Spec}(\mathbb{C})$ ).

(d) The representations $\mathcal{O}_{X}[G / H]$ considered in the above corollaries are already defined over $\mathbb{Z}$. This implies in particular that, for any separably closed field $L$ with prime field $K$, the representations $L[G / H]$ are invariant under the Galois group $\operatorname{Gal}(L / K)$. Then, by functoriality, the same holds for the Chern classes of $L[G / H]$. This fact was used by Grothendieck (see $\S 4$ of [Gro2]) to construct universal annihilators for these Chern classes. This method is applicable not only to induced representations but to arbitrary representations with known isotropy group and it yields better annihilators. However, the annihilators presented here are annihilators for the Chern classes over the prime field and even over the integers $\mathbb{Z}$.

(e) Thomas has translated the invariance of complex representations under the Galois group $\operatorname{Gal}(\mathbb{C} / K)$ of $\mathbb{C}$ over a number field $K$ into the invariance of these representations in $K_{0}(G, \mathbb{C})$ under the Adams operations for certain $j$. Similarly to Corollary (6.11), he then constructs annihilators for the transcendental Chern classes of these representations in group cohomology $H^{*}(G, \mathbb{Z})$ (see [Tho], p. 55 and pp. 81-84).

(f) For elements of $K(G, X)$ or $\hat{K}(G, X)\left[j^{-1}\right]$ which are eigenvectors of $\psi^{j}$ with an integral eigenvalue, which is different from 1 , say $j^{N}$ for some $N$, the method used in the proof of Corollary (6.11) would yield similarly defined universal annihilators for the associated Chern classes. I do not know whether it is reasonable to expect that such elements exist apart from those which are induced from the non-equivariant part of $K(H, X)$ for some subgroup $H$ of $G$. For instance, all elements of the representation $\operatorname{ring} K_{0}(G, \mathbb{Q})$ of a finite abelian group $G$ are invariant under $\psi^{j}$ in $\hat{K}_{0}(G, \mathbb{Q})\left[j^{-1}\right]$ since $K_{0}(G, \mathbb{Q})$ is generated by induced representations. Example (6.6) presents a case where eigenvalues different from 1 occur but these are not integral. 


\section{Higher $T$-Equivariant Algebraic $K$-Theory of $G / B$}

In this section, let $S$ be a separated regular noetherian connected scheme, $G$ a simply connected split semisimple group scheme over $S$ of rank $l, T$ a split maximal torus in $G$, and $B$ a Borel subgroup of $G$ containing $T$.

Modifying and combining some arguments contained in the paper [KK2] of Kostant and Kumar, we establish an isomorphism between the higher $T$-equivariant algebraic $K$-theory $K(T, G / B)$ of $G / B$ and the ring $K(S) \otimes \Psi$ where $\Psi$ is a certain combinatorically defined ring introduced in [KK2]. From this isomorphism, we deduce that $K(T, G / B)$ is a $\lambda$-ring as conjectured in (2.7) and that the push-forward homomorphism $\pi_{*}: K(T, G / B) \rightarrow K(T, S)$ associated with the structure morphism $\pi: G / B \rightarrow S$ is continuous with respect to the Grothendieck filtrations as conjectured in (5.6). Applying the equivariant Grothendieck-Riemann-Roch theorem to $\pi$, we finally give a new proof of the Weyl character formula.

First, we recall the definition of the ring $\Psi$. For this, we introduce the following notations. Let $W$ be the Weyl group of $(G, T)$ considered as the set of $S$-valued points of $\mathrm{N}_{G}(T) / T$. It acts on the character group $X(T):=\operatorname{Hom}_{S}\left(T, \mathbf{G}_{m}\right)$ as usual by $(w \cdot \lambda)(t):=\lambda\left(w^{-1} t w\right)$ for $\lambda \in X(T)$, $w \in W$ and $t \in T$. Let $R \subset X(T)$ be the associated root system, and let $\alpha_{1}, \ldots, \alpha_{l} \in R$ be the simple roots associated with $(G, B, T)$. (The convention used here is that the root spaces corresponding to positive roots are contained in the Lie algebra of $B$.) Let $r_{1}, \ldots, r_{l} \in W$ denote the associated simple reflections, $\rho_{1}, \ldots, \rho_{l} \in X(T)$ the associated fundamental weights (uniquely determined by $r_{j} \rho_{i}=\rho_{i}-\delta_{i, j} \alpha_{j}$ for all $i, j=1, \ldots, l$ ), and $P_{1}, \ldots, P_{l}$ the associated minimal standard parabolic subgroups of $G$ (given by $P_{i}=B \cup B r_{i} B$ for all $i=1, \ldots l$ ). The group ring $\mathbb{Z}[X(T)]$ is denoted by $R(T)$, and the canonical basis elements in $R(T)$ are denoted by $e^{\lambda}, \lambda \in X(T)$. By Example (2.8)(d), we have a canonical ring isomorphism

$$
K(S) \otimes R(T) \cong K(T, S) .
$$

By $Q(T)$ we denote the quotient field of $R(T)$. The action of $W$ on $X(T)$ induces an action of $W$ on $R(T)$ and $Q(T)$. Let $Q(T)_{W}:=Q(T) \# W$ denote the associated twisted group ring. The canonical basis elements in $Q(T)_{W}$ are denoted by $\delta_{w}, w \in W$. The multiplication in $Q(T)_{W}$ is normalized by $\delta_{w} \cdot q=(w q) \cdot \delta_{w}$ for $q \in Q(T)$ and $w \in W$. For any $i=1, \ldots, l$, let

$$
y_{i}:=y_{r_{i}}:=\left(\delta_{e}+\delta_{r_{i}}\right) \frac{1}{1-e^{\alpha_{i}}}=\frac{1}{1-e^{\alpha_{i}}}\left(\delta_{e}-e^{\alpha_{i}} \delta_{r_{i}}\right) \in Q(T)_{W} .
$$

(Compared with [KK2], we have changed this definition and some other definitions introduced later on in order to avoid the anti-automorphism ${ }^{t}$ of $Q(T)_{W}$ and various inversions occurring in [KK2].) For any $w \in W$, we put

$$
y_{w}:=y_{i_{1}} \cdot \ldots \cdot y_{i_{l(w)}} \in Q(T)_{W}
$$

where $w=r_{i_{1}} \ldots \cdot r_{i_{l(w)}}$ is a reduced decomposition of $w \in W$. This definition does not depend on the chosen decomposition of $w$ by Proposition (2.4) of [KK2]. We consider $Q(T)$ as a left $Q(T)_{W}$-module via $\left(\delta_{w} q\right) \cdot q^{\prime}=w\left(q \cdot q^{\prime}\right) \Leftrightarrow\left(q \delta_{w}\right) \cdot q^{\prime}=q \cdot w\left(q^{\prime}\right)$ for $q, q^{\prime} \in Q(T)$ and $w \in W$, and we put

$$
Y:=\left\{y \in Q(T)_{W}: y \cdot R(T) \subset R(T)\right\} \subseteq Q(T)_{W} .
$$

Obviously, $Y$ is a subring of $Q(T)_{W}$ with $R(T) \# W \stackrel{\text { def }}{=} \sum R(T) \delta_{w}=\sum \delta_{w} R(T) \subseteq Y$ and it is an $R(T)$-submodule of $Q(T)_{W}$ with respect to both left and right multiplication. Furthermore, 
the elements $y_{w}, w \in W$, are contained in $Y$ since, for all $i=1, \ldots, l$ and $\lambda \in X(T)$, we have $r_{i} \lambda=\lambda-n_{i}(\lambda) \alpha_{i}$ with some $n_{i}(\lambda) \in \mathbb{Z}$.

(7.1) Proposition. The elements $y_{w}, w \in W$, form a basis of the $R(T)$-module $Y$ (with respect to both left and right multiplication).

Proof. See Theorem (2.9) of [KK2].

Now, we consider $Y$ as an $R(T)$-module via left multiplication and define

$$
\Psi:=\operatorname{Hom}_{R(T)}(Y, R(T))
$$

to be the dual of $Y$. The dual basis of $\Psi$ associated with $y_{w}, w \in W$, is denoted by $\psi^{w}$, $w \in W$.

(7.2) Lemma. For any commutative ring $K$, the $K$-linear map

$$
\begin{aligned}
K \otimes \Psi & \rightarrow K \otimes \operatorname{Hom}_{R(T)}(R(T) \# W, R(T))=\operatorname{Maps}(W, K \otimes R(T)) \\
\psi & \left.\mapsto \psi\right|_{R(T) \# W}
\end{aligned}
$$

is injective.

Proof. Let $Q$ be the quotient $Y / R(T) \# W$. Since the sequence

$$
0 \rightarrow \operatorname{Hom}_{R(T)}(Q, K \otimes R(T)) \rightarrow \operatorname{Hom}_{R(T)}(Y, K \otimes R(T)) \rightarrow \operatorname{Hom}_{R(T)}(R(T) \# W, K \otimes R(T))
$$

is exact, we have to show that $\operatorname{Hom}_{R(T)}(Q, K \otimes R(T))$ vanishes. Let $\alpha \in \operatorname{Hom}_{R(T)}(Q, K \otimes R(T))$ and $q \in Q$. By Proposition (7.1), there are roots $\beta_{i} \in R$ such that $r=\prod_{i}\left(1-e^{\beta_{i}}\right)$ annihilates $q$. Then we also have $r \cdot \alpha(q)=0$ in $K \otimes R(T)$. Since $r$ contains no prime factor coming from $\mathbb{Z}$, the factor ring $R(T) /(r)$ is torsion free, hence flat over $\mathbb{Z}$. Thus, the multiplication with $r$ is universally injective. Hence, we have $\alpha(q)=0$ as was to be shown.

We consider the $R(T)$-module $\operatorname{Maps}(W, R(T))$ as an $R(T)$-algebra with multiplication defined pointwise. Then we have:

(7.3) Proposition. The $R(T)$-module $\Psi$ is an $R(T)$-subalgebra of $\operatorname{Maps}(W, R(T))$ via the embedding defined in Lemma (7.2).

Proof. See Proposition (2.20)(a) of [KK2].

Now, we are going to identify the higher $T$-equivariant algebraic $K$-theory $K(T, G / B)$ of $G / B$ with $K(S) \otimes \Psi$. Recall that $T$ acts on $G / B$ via left multiplication and that the set $(G / B)^{T}$ of ( $S$-valued) fixed points can be identified with $W$ via $w \mapsto w B / B$. Let $\bar{\gamma}$ be the localization map

$$
\bar{\gamma}: K(T, G / B) \stackrel{i^{*}}{\rightarrow} K\left(T,(G / B)^{T}\right)=\operatorname{Maps}(W, K(T, S))=\operatorname{Maps}(W, K(S) \otimes R(T)) .
$$

Here, $i:(G / B)^{T} \hookrightarrow G / B$ denotes the canonical inclusion. Obviously, $\bar{\gamma}$ is a homomorphism of $K(T, S)$-algebras.

(7.4) Theorem. The map $\bar{\gamma}$ induces an isomorphism

$$
\gamma: K(T, G / B) \stackrel{\sim}{\rightarrow} K(S) \otimes \Psi
$$

of $K(T, G / B)$ with the $K(T, S)$-subalgebra $K(S) \otimes \Psi$ of $\operatorname{Maps}(W, K(S) \otimes R(T)$ ) (cf. Lemma (7.2) and Proposition (7.3)). 
Proof. This immediately follows from Proposition (7.1) and the following three propositions. Here, the injectivity of $\gamma$ follows from the surjectivity of $\gamma$ and Propositions (7.1) and (7.5). (Alternatively, this can be deduced from the localization theorem, see last assertion of Lemme 3.3 in [Th4] and the proof of Lemma (7.2).)

(7.5) Proposition. The $K(T, S)$-module $K(T, G / B)$ is free of rank $|W|$.

(7.6) Proposition. The image of $\bar{\gamma}$ is contained in $K(S) \otimes \Psi$.

(7.7) Proposition. The image of $\bar{\gamma}$ contains $K(S) \otimes \Psi$.

Proof (of Proposition (7.5)). We recall the following standard arguments (e. g. see the appendix of [Ko1]). By Corollary 5.8 (3) of [Th3], we have $K(T, G / B) \cong K^{\prime}(T, G / B)$ where $K^{\prime}(T, G / B)$ denotes the higher $K$-theory associated with the category of coherent $T$-modules on $G / B$. Furthermore, the Bruhat decomposition of $G / B$ yields a filtration

$$
S=Y_{0} \subset Y_{1} \subset \ldots \subset Y_{|W|}=G / B
$$

of $G / B$ by $T$-stable closed $S$-subschemes of $G / B$ such that for all $i$ the canonical projection $p_{i}: Y_{i} \backslash Y_{i-1} \rightarrow S$ is $T$-isomorphic to an affine space $\mathbb{A}_{S}^{n_{i}}$ with linear $T$-action. Hence, by the equivariant homotopy theorem (see Theorem 4.1 of [Th3]), the pull-back homomorphism $p_{i}^{*}: K^{\prime}(T, S) \rightarrow K^{\prime}\left(T, Y_{i} \backslash Y_{i-1}\right)$ is an isomorphism for all $i$. Therefore, by the equivariant localization theorem (see Theorem 2.7 of [Th3]), we have split short exact sequences

$$
0 \rightarrow K_{q}^{\prime}\left(T, Y_{i-1}\right) \rightarrow K_{q}^{\prime}\left(T, Y_{i}\right) \rightarrow K_{q}^{\prime}\left(T, Y_{i} \backslash Y_{i-1}\right) \rightarrow 0, \quad q \geq 0,
$$

for $i=1, \ldots,|W|$. Now induction yields

$$
K(T, G / B) \cong K^{\prime}(T, G / B) \cong \operatorname{Maps}\left(W, K^{\prime}(T, S)\right) \cong \operatorname{Maps}(W, K(T, S))
$$

as was to be shown.

In order to prove Proposition (7.6), we first introduce a further notation and prove a preparatory lemma: Any character $\lambda: T \rightarrow \mathbf{G}_{m}$ canonically induces a representation $\lambda: B \stackrel{\text { can }}{\longrightarrow} T \stackrel{\lambda}{\longrightarrow}$ $\mathbf{G}_{m}$ of $B$ of rank 1 whose underlying representation space we denote by $V(\lambda)$. Then the locally free $T$-module $\mathcal{L}(\lambda)$ on $G / B$ is defined to be the sheaf of sections of the $T$-vectorbundle $G \times{ }^{B} V(\lambda)$ over $G / B$ : For any open subset $U$ of $G / B$, we have

$$
\Gamma(U, \mathcal{L}(\lambda))=\left\{\phi: \pi_{B}^{-1}(U) \rightarrow V(\lambda) S \text {-morphism }: \phi(x b)=\lambda(b)^{-1} \phi(x) \text { for } x \in \pi_{B}^{-1}(U), b \in B\right\}
$$

where $\pi_{B}: G \rightarrow G / B$ denotes the canonical projection. Similarly, the $T$-module $\mathcal{L}_{0}(\lambda)$ on $P_{i} / B$ is defined. (See section 5, especially section 5.16, of part I and section (1.10) of part II of $[\mathrm{J}]$ for this definition.)

For any $i=1, \ldots, l$ let $\pi_{i}: G / B \rightarrow G / P_{i}$ denote the canonical projection $\left(P_{i}=B \cup B r_{i} B\right)$.

(7.8) Lemma. The $T$-module $\mathcal{E}:=\left(\pi_{i}\right)_{*}\left(\mathcal{L}\left(-\rho_{i}\right)\right)$ on $G / P_{i}$ is locally free of rank 2 , the adjunction homomorphism $\pi_{i}^{*} \mathcal{E} \rightarrow \mathcal{L}\left(-\rho_{i}\right)$ is surjective, and the induced $T$-morphism

$$
G / B \rightarrow \mathbb{P}_{G / P_{i}}(\mathcal{E})
$$

of $G / P_{i}$-schemes (see (1.5)) is an isomorphism.

Proof. By assertion (5) of $[\mathrm{J}]$ on p. 183, there is a cover of $G / P_{i}$ by open subsets $U$ which have the following property: There is an isomorphism between $\pi_{i}^{-1}(U)$ and $U \times P_{i} / B$ such that the 
diagram

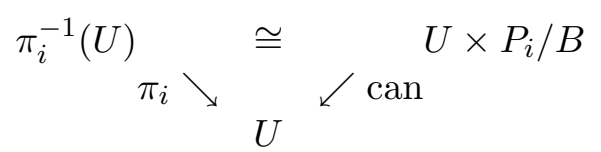

commutes and such that the $T$-module $\left.\mathcal{L}\left(-\rho_{i}\right)\right|_{\pi_{i}^{-1}(U)}$ on $\pi_{i}^{-1}(U)$ corresponds to the vector bundle $U \times P_{i} \times{ }^{B} V(\lambda)$ on $U \times P_{i} / B$. Therefore, by the usual base change argument (see Corollaire (9.3.3) of [EGA] I), it suffices to show the following assertions for the $T$-morphism $q_{i}: P_{i} / B \rightarrow S:$ The $T$-module $\mathcal{E}_{0}:=\left(q_{i}\right)_{*}\left(\mathcal{L}_{0}\left(-\rho_{i}\right)\right)$ is isomorphic to $\mathcal{O}_{S}^{2}$, the adjunction homomorphism $q_{i}^{*} \mathcal{E}_{0} \rightarrow \mathcal{L}_{0}\left(-\rho_{i}\right)$ is surjective, and the induced $T$-morphism

$$
P_{i} / B \rightarrow \mathbb{P}_{S}\left(\mathcal{E}_{0}\right)=\mathbb{P}_{S}^{1}
$$

of $S$-schemes is an isomorphism. Using a Levi decomposition of $P_{i}$, we may furthermore assume that $P_{i}$ is reductive of semisimple rank 1. Then, by Proposition (5.8)(i) in Exp. XX of [SGA3], we are reduced to $P_{i}=\mathrm{SL}_{2}$. Under this reduction, $-\rho_{i}$ corresponds to the character $\left(\begin{array}{cc}z & 0 \\ 0 & z^{-1}\end{array}\right) \mapsto\left(-\rho_{i}\right) \circ \alpha_{i}^{\vee}(z)$ where $\alpha_{i}^{\vee}: \mathbf{G}_{m} \rightarrow T$ denotes the coroot associated with $\alpha_{i}$ (loc. cit.). But $\rho_{i} \circ \alpha_{i}^{\vee}$ is the identity character since $r_{i} \rho_{i}=\rho_{i}-\alpha_{i}$. Now, an easy computation shows that $\mathcal{L}\left(-\rho_{i}\right)$ corresponds to the twisting sheaf $\mathcal{O}(1)$ under the isomorphism $P_{i} / B \cong \mathbb{P}_{S}^{1}$ as was to be shown.

Proof (of Proposition (7.6)). For any $i=1, \ldots, l$, let $D_{i}$ denote the $K(T, S)$-linear map $D_{i}:=\pi_{i}^{*} \circ\left(\pi_{i}\right)_{*}: K(T, G / B) \rightarrow K(T, G / B)$. We introduce the structure of a left $Y$-module on $\Psi$ and on $\Omega:=\operatorname{Hom}_{Q(T)}\left(Q(T)_{W}, Q(T)\right)$ as follows:

$$
(y \cdot \psi)\left(y^{\prime}\right):=\psi\left(y^{\prime} \cdot y\right) \quad \text { for } \quad \psi \in \Psi \text { or } \Omega \quad \text { and } \quad y, y^{\prime} \in Y \text { or } Q(T)_{W} .
$$

Then we claim that we have

$$
\bar{\gamma}\left(D_{i}(\tau)\right)=y_{i} \cdot \bar{\gamma}(\tau) \quad \text { in } \quad \Omega \quad \text { for all } \quad \tau \in K(T, G / B) .
$$

To give sense to this equality, we identify $\bar{\gamma}(\tau) \in K(S) \otimes \operatorname{Hom}_{R(T)}(R(T) \# W, R(T))$ with its image in $K(S) \otimes \Omega$. (Here, one should actually replace $Q(T)$ by the localization of $R(T)$ with respect to the multiplicative submonoid of $R(T)$ generated by the elements $1-e^{\alpha}, \alpha \in R$. Then the canonical injection $\Psi \hookrightarrow \operatorname{Hom}_{Q(T)}\left(Q(T)_{W}, Q(T)\right)$ remains injective after tensoring with $K(S)$ by the proof of Lemma (7.2).) Having proved this claim, we know that, for all $w \in W$, the element $\bar{\gamma}(\tau)\left(y_{w}\right)=\left(y_{w} \cdot \bar{\gamma}(\tau)\right)\left(\delta_{e}\right)$ is contained in $K(T, S)$. Thus, by Proposition (7.1), $\bar{\gamma}(\tau)$ is an element of $\Psi$ as was to be shown.

In order to prove the above claim, we first consider a $\tau \in K(T, G / B)$ which is contained in the image of $\pi_{i}^{*}$ and put $\psi:=\bar{\gamma}(\tau) \in \Omega$. Since the diagram

$$
\begin{array}{crr}
K\left(T, G / P_{i}\right) & \stackrel{i_{p_{i}}^{*}}{\longrightarrow} & K\left(T,\left(G / P_{i}\right)^{T}\right) \\
\downarrow \pi_{i}^{*} & & \downarrow\left(\pi_{i}^{T}\right)^{*} \\
K(T, G / B) & \stackrel{i^{*}}{\longrightarrow} & K\left(T,(G / B)^{T}\right)
\end{array}
$$

commutes we have $\psi(w)=\psi\left(w r_{i}\right)$ for all $w \in W$. Hence, we have

$$
\left(y_{r_{i}} \cdot\left(\psi \psi^{\prime}\right)\right)(w)=\left(\psi \psi^{\prime}\right)\left(\delta_{w} \cdot y_{r_{i}}\right)
$$




$$
\begin{aligned}
& =\left(\psi \psi^{\prime}\right)\left(\frac{1}{1-e^{w \alpha_{i}}}\left(\delta_{w}-e^{w \alpha_{i}} \delta_{w r_{i}}\right)\right) \\
& =\psi\left(\delta_{w}\right) \cdot \frac{1}{1-e^{w \alpha_{i}}}\left(\psi^{\prime}\left(\delta_{w}\right)-e^{w \alpha_{i}} \psi^{\prime}\left(\delta_{w r_{i}}\right)\right) \\
& =\left(\psi \cdot\left(y_{r_{i}} \cdot \psi^{\prime}\right)\right)(w) .
\end{aligned}
$$

for all $\psi^{\prime} \in \Omega$ and $w \in W$. This means that the multiplication with $y_{r_{i}}$ on $\Omega$ is linear over $\bar{\gamma}\left(\operatorname{Image}\left(\pi_{i}^{*}\right)\right)$. Since, on the other hand, $D_{i}$ is linear over Image $\left(\pi_{i}^{*}\right)$, and since, by Lemma (7.8) and the equivariant projective space bundle theorem $(2.2), K(T, G / B)$ is a free $K\left(T, G / P_{i}\right)$ module with basis $1, \mathcal{L}\left(\rho_{i}\right)$ it suffices to show the above claim for $\tau=1$ and $\tau=\mathcal{L}\left(\rho_{i}\right)$. We obviously have $\bar{\gamma}\left(D_{i}(1)\right)=1=y_{r_{i}}(\bar{\gamma}(1))$. This shows the above claim for $\tau=1$. Furthermore, an easy computation shows that $\bar{\gamma}\left(\mathcal{L}\left(\rho_{i}\right)\right)\left(\delta_{w}\right)=e^{w \rho_{i}}$ for all $w \in W$. Hence, we have

$$
\begin{gathered}
\left(y_{r_{i}} \cdot \bar{\gamma}\left(\mathcal{L}\left(\rho_{i}\right)\right)\right)\left(\delta_{w}\right)=\bar{\gamma}\left(\mathcal{L}\left(\rho_{i}\right)\right)\left(\frac{1}{1-e^{w \alpha_{i}}}\left(\delta_{w}-e^{w \alpha_{i}} \delta_{w r_{i}}\right)\right) \\
=\frac{1}{1-e^{w \alpha_{i}}}\left(e^{w \rho_{i}}-e^{w \alpha_{i}} e^{w r_{i} \rho_{i}}\right)=0
\end{gathered}
$$

for all $w \in W$ since $r_{i} \rho_{i}=\rho_{i}-\alpha_{i}$. Since $D_{i}\left(\mathcal{L}\left(\rho_{i}\right)\right)$ is zero (see section 3 ), we have $\bar{\gamma}\left(D_{i}\left(\mathcal{L}\left(\rho_{i}\right)\right)\right)=0=y_{r_{i}} \cdot \bar{\gamma}\left(\mathcal{L}\left(\rho_{i}\right)\right)$ as was to be shown.

Proof (of Proposition (7.7)). Let $\beta: R(T) \rightarrow K_{0}(T, G / B)$ denote the Atiyah-Hirzebruch homomorphism (given by $e^{\lambda} \mapsto \mathcal{L}(\lambda)$ ), and let $\phi$ denote the $K(T, S)$-linear homomorphism

$$
\phi: K(T, S) \otimes R(T) \rightarrow K(T, G / B), \quad f \otimes g \mapsto f \cdot \beta(g) .
$$

Similarly, let $\bar{\phi}$ denote the $K(T, S)$-linear homomorphism

$$
\bar{\phi}: K(T, S) \otimes R(T) \rightarrow K(S) \otimes \Psi, \quad 1 \otimes e^{\lambda} \mapsto 1 \otimes e^{\lambda} \cdot 1 \hat{=} 1 \otimes\left(w \mapsto e^{w \lambda}\right) .
$$

Then the diagram

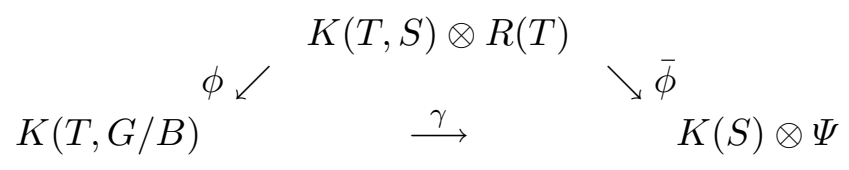

obviously commutes. Since, by the proof of Theorem (4.4) in [KK2], the map $\bar{\phi}$ is surjective, also $\gamma$ is surjective. This proves Proposition (7.7).

(7.9) Corollary. The pre- $\lambda$-ring $K(T, G / B)$ is a $\lambda$-ring, i. e. Conjecture (2.7) is true for the $T$-scheme $X:=G / B$.

Proof. By Remark (2.8)(a) and Lemma (2.4), we know that $K(S)$ and $R(T)$ are $\lambda$-rings. Then, by Lemma (6.2) of $[\mathrm{AT}]$, also $K(S) \otimes R(T) \otimes R(T)=K(T, S) \otimes R(T)$ is a $\lambda$-ring. Here, the $\lambda$-structure is defined using the universal polynomials $P_{n}, n \geq 1$, defined e. g. on page 5 of $[\mathrm{FL}]$. Since, by Theorem (7.4) and the proof of Proposition (7.7), the homomorphism $\phi: K(T, S) \otimes R(T) \rightarrow K(T, G / B)$ is a surjective ring homomorphism which by Proposition (2.5) is compatible with the $\lambda$-structures, Corollary (7.9) follows from this.

(7.10) Corollary. The $\left(F^{n}\right)$-topology on $K(T, G / B)$ agrees with that topology on $K(T, G / B)$ which is induced from the $\left(F^{n}\right)_{n \geq 0}$-topology on $K(T, S)$ by the pull-back homomorphism $\pi^{*}$. In particular, Conjecture (5.6) is true for the $T$-morphism $\pi: G / B \rightarrow S$. 
Proof. Since, by Theorem (7.4) and Corollary (7.9), we have $\lambda$-ring isomorphisms $K(T, G / B) \cong$ $K(S) \otimes \Psi$ and $K(T, S) \cong K(S) \otimes R(T)$, it suffices to show the corresponding assertion for $K_{0}$ in the case $S=\operatorname{Spec}(\mathbb{C})$.

Let $I$ be the augmentation ideal of $R(T)$. By (the proof of) of Proposition (5.1), the Grothendieck filtration on $R(T)$ agrees with the the $I$-adic filtration. Because of the surjectivity of the $\lambda$-ring homomorphism $\phi: R(T) \otimes R(T) \rightarrow K_{0}(T, G / B)$, also the Grothendieck filtration on $K_{0}(T, G / B)$ agrees with the $F_{0}^{1}(T, G / B)$-adic filtration. Hence, Corollary (7.10) follows from the following inclusions:

$$
F_{0}^{1}(T, G / B)^{l|W|} \subseteq I K_{0}(T, G / B) \subseteq F_{0}^{1}(T, G / B) .
$$

Here, the second inclusion is trivial and the proof of the first inclusion runs as follows.

Obviously, we have $\bar{\phi}\left(\prod_{w \in W}\left(1 \otimes e^{\lambda}-e^{w \lambda} \otimes 1\right)\right)=0$ in $\Psi \subset \operatorname{Maps}(W, R(T))$ for all $\lambda \in X(T)$. Hence, by Theorem (7.4) and the proof of Proposition (7.7), the element

$$
\prod_{w \in W}\left((\mathcal{L}(\lambda)-1)+\left(1-e^{w \lambda}\right)\right)=\prod_{w \in W}\left(\mathcal{L}(\lambda)-e^{w \lambda}\right) \quad \text { of } \quad K_{0}(T, G / B)
$$

vanishes for all $\lambda \in X(T)$. This shows that $(\mathcal{L}(\lambda)-1)^{|W|}$ is contained in $I K_{0}(T, G / B)$ for all $\lambda \in X(T)$. Since the ideal $I$ is generated by $l$ elements of the form $\mathcal{L}(\lambda)-1$, this shows the first inclusion in the above claim.

Conjecture (5.6) follows from this like in the proof of Proposition (5.8).

Next, we show how the famous Weyl character formula can be deduced from the equivariant Grothendieck-Riemann-Roch theorem (5.10) for $K_{0}$ applied to $\pi$. Note that this theorem is available now by Corollary (7.10) but we will show the continuity of $\pi_{*}$ again proving the stronger inclusion $F_{0}^{1}(T, G / B)^{|R|} \subseteq I K_{0}(T, G / B)$. Certainly, the Lefschetz fixed point formula is the most natural approach to prove the Weyl character formula. So the following considerations should perhaps be regarded only as an example making explicit all the terms we have introduced in the previous sections.

The canonical projection $\pi: G / B \rightarrow S$ is a projective, smooth $T$-morphism and hence a $T$ projective, local complete intersection morphism by Remark (3.5). Let $\rho \in X(T)$ denote the half sum of the positive roots, and, for any $\lambda \in X(T)$, let $A(\lambda):=\sum_{w \in W} \operatorname{det}(w) e^{w \lambda} \in R(T) \subseteq$ $K_{0}(T, S)$.

(7.11) Theorem (Weyl character formula). For all $\lambda \in X(T)$, we have

$$
\pi_{*}(\mathcal{L}(\lambda))=\frac{A(\lambda-\rho)}{A(-\rho)} \quad \text { in } \quad K_{0}(T, S)
$$

Proof. We may assume that $S=\operatorname{Spec}(\mathbb{Z})$. Then we have $K_{0}(T, S)=R(T)$. We define an $R(T)$-linear map

$$
D: K_{0}(T, G / B) \rightarrow R(T)
$$

as follows: Let $D: K_{0}(T, G / B) \rightarrow K_{0}(T, G / B)$ be the composition of the maps $D_{i_{k}}, k=$ $1, \ldots, l\left(w_{0}\right)$, (see the proof of Proposition (7.6)) where $w_{0}=r_{i_{1}} \cdot \ldots \cdot r_{i_{l(w)}}$ is a reduced decomposition of the longest element $w_{0} \in W$. As shown in (7.6), it corresponds to the operator $y_{w_{0}}$ via the isomorphism $\gamma$. Hence, by section 5.6 of [De], we have

$$
D(\mathcal{L}(\lambda))=\frac{A(\lambda-\rho)}{A(-\rho)}=\sum_{w \in W}\left(e^{\lambda} \cdot \frac{e^{-\rho}}{A(-\rho)}\right)^{w}
$$


for all $\lambda \in X(T)$. In particular, the image of $D$ is contained in $R(T) \subseteq K_{0}(T, G / B)$ since $K_{0}(T, G / B)$ is generated by the elements $\mathcal{L}(\lambda), \lambda \in X(T)$, as $R(T)$-module. Because of $\frac{e^{-\rho}}{A(-\rho)}=\prod_{\alpha \in R_{+}} \frac{1}{1-e^{\alpha}}$ (see Lemma (24.3) of $[\mathrm{FH}]$ ), we have

$$
D(\phi(1 \otimes f))=\sum_{w \in W}\left(f \cdot \prod_{\alpha \in R_{+}} \frac{1}{1-e^{\alpha}}\right)^{w}
$$

for all $f \in R(T)$. We have to show that $\pi_{*}=D$.

As seen in the proof of Corollary (7.10), the Grothendieck filtration on $K_{0}(T, G / B)$ agrees with the $F_{0}^{1}(T, G / B)$-adic filtration. Furthermore, the ideal $F_{0}^{1}(T, G / B)$ obviously corresponds to the ideal $F^{1}(\Psi):=\Psi \cap \operatorname{Maps}(W, I)$ of $\Psi$ via the isomorphism $\gamma$. More generally, the ideal $F_{0}(T, G / B)^{n}$ being the image of $(R(T) \otimes I+I \otimes R(T))^{n}$ under $\phi$ corresponds to the ideal $F^{n}(\Psi):=\Psi \cap \operatorname{Maps}\left(W, I^{n}\right)$ for all $n \geq 0$. By Proposition (2.22)(e) and the proof of Lemma (2.29) in [KK2], we have

$$
F^{n}(\Psi)=\underset{w \in W}{\oplus} I^{n-l(w)} \psi^{w}
$$

where $\psi^{w} \in \Psi, w \in W$, is the dual basis of $y_{w} \in Y, w \in W$. Hence, the associated completed graded ring $\hat{\operatorname{Gr}}(\Psi)$ is a free $\hat{\operatorname{Gr}} R(T)$-module with basis $\overline{\psi^{w}} \in \mathrm{Gr}^{l(w)} \Psi, w \in W$. Furthermore, by Proposition (2.30) of [KK2], $\operatorname{Gr}(\Psi)_{\mathbb{C}}$ is graded isomorphic to the ring $\Lambda$ defined in [KK1]. Using the operators $A_{w}, w \in W$ defined in $\S 4$ of [KK1], one similarly constructs a $\hat{\operatorname{Gr}} R(T)_{\mathbb{Q}^{-}}$ linear map

$$
L: \hat{\operatorname{Gr}} K_{0}(T, G / B)_{\mathbb{Q}} \rightarrow \hat{\operatorname{Gr}} R(T)_{\mathbb{Q}}
$$

such that for all $g \in \hat{\mathrm{Gr}} R(T)_{\mathbb{Q}}$ we have

$$
L(\hat{\operatorname{Gr}}(\phi)(1 \otimes g))=\sum_{w \in W}\left(g \cdot \prod_{\alpha \in R_{+}} \frac{1}{c_{1}\left(e^{-\alpha}\right)}\right)^{w} \quad \text { in } \quad \hat{\mathrm{Gr}} R(T)_{\mathbb{Q}} .
$$

Here, $\hat{\operatorname{Gr}}(\phi): \hat{\operatorname{Gr}} R(T) \otimes \hat{\mathrm{Gr}} R(T) \rightarrow \hat{\mathrm{Gr}} K_{0}(T, G / B)$ denotes the graded version of $\phi$ and $c_{1}\left(e^{-\alpha}\right)=\left(e^{-\alpha}-1\right)+I^{2} \in \operatorname{Gr}^{1} R(T)$ denotes the first Chern class of $e^{-\alpha}$.

By the equivariant Grothendieck-Riemann-Roch theorem (5.10), the diagram

$$
\begin{array}{crr}
K_{0}(T, G / B) & \stackrel{\operatorname{Td}\left(T_{\pi}\right) \cdot \operatorname{ch}}{\longrightarrow} & \hat{\operatorname{Gr}} K_{0}(T, G / B)_{\mathbb{Q}} \\
\downarrow \pi_{*} & & \downarrow \hat{\mathrm{Gr}}\left(\pi_{*}\right)_{\mathbb{Q}} \\
R(T) & \stackrel{\mathrm{ch}}{\longrightarrow} & \hat{\mathrm{Gr}} R(T)_{\mathbb{Q}}
\end{array}
$$

commutes. Here, we have

$$
\operatorname{Td}\left(T_{\pi}\right)=\prod_{\alpha \in R_{+}} \frac{c_{1}(\mathcal{L}(-\alpha))}{1-\exp \left(c_{1}(\mathcal{L}(\alpha))\right)}
$$

since $T_{\pi}=\sum_{\alpha \in R_{+}} \mathcal{L}(-\alpha)$ in $K_{0}(T, G / B)$ by formula (4) of [J] on p. 229. The following formal computation (analogous to $\S 4$ of $[\mathrm{La}]$ ) shows that also the diagram

$$
\begin{array}{ccc}
K_{0}(T, G / B) & \stackrel{\operatorname{Td}\left(T_{\pi}\right) \cdot \operatorname{ch}}{\longrightarrow} & \hat{\operatorname{Gr}} K_{0}(T, G / B)_{\mathbb{Q}} \\
\downarrow D & & \downarrow L \\
R(T) & \stackrel{\mathrm{ch}}{\longrightarrow} & \hat{\mathrm{Gr}} R(T)_{\mathbb{Q}}
\end{array}
$$


commutes:

$$
\begin{aligned}
\operatorname{ch} D & (\mathcal{L}(\lambda))=\operatorname{ch} \sum_{w \in W}\left(e^{\lambda} \cdot \prod_{\alpha \in R_{+}} \frac{1}{1-e^{\alpha}}\right)^{w} \\
& =\sum_{w \in W}\left(\exp \left(c_{1}\left(e^{\lambda}\right)\right) \cdot \prod_{\alpha \in R_{+}} \frac{1}{1-\exp \left(c_{1}\left(e^{\alpha}\right)\right)}\right)^{w} \\
& =\sum_{w \in W}\left(\exp \left(c_{1}\left(e^{\lambda}\right)\right) \cdot \prod_{\alpha \in R_{+}} \frac{c_{1}\left(e^{-\alpha}\right)}{1-\exp \left(c_{1}\left(e^{\alpha}\right)\right)} \cdot \prod_{\alpha \in R_{+}} \frac{1}{c_{1}\left(e^{-\alpha}\right)}\right)^{w} \\
& =L\left(\exp \left(c_{1}(\mathcal{L}(\lambda))\right) \cdot \prod_{\alpha \in R_{+}} \frac{c_{1}(\mathcal{L}(-\alpha))}{1-\exp \left(c_{1}(\mathcal{L}(\alpha))\right)}\right) \\
& =L\left(\operatorname{ch}(\mathcal{L}(\lambda)) \cdot \operatorname{Td}\left(T_{\pi}\right)\right) .
\end{aligned}
$$

By Example (5.5), the Chern character ch $: R(T) \rightarrow \hat{\mathrm{Gr}} R(T)_{\mathbb{Q}}$ is injective. Thus, in order to show $D=\pi_{*}$, it suffices to show that $L=\hat{\operatorname{Gr}}\left(\pi_{*}\right)_{\mathbb{Q}}$. For this, we identify the basis elements $\psi^{w}, w \in W$, of $\Psi$ with their preimages in $K_{0}(T, G / B)$ under $\gamma$. Since their residue classes form a basis for the associated completed graded object, and since both $L$ and $\hat{\operatorname{Gr}}\left(\pi_{*}\right)_{\mathbb{Q}}$ are $\hat{\operatorname{Gr}} R(T)_{\mathbb{Q}}$-linear of degree $-l\left(w_{0}\right)$, it suffices to show the equality

$$
\operatorname{rank}\left(\pi_{*}\left(\psi^{w_{0}}\right)\right) \stackrel{\text { def }}{=} \hat{\operatorname{Gr}}\left(\pi_{*}\right)_{\mathbb{Q}}\left(\overline{\psi^{w_{0}}}\right)=L\left(\overline{\psi^{w_{0}}}\right) \quad \text { in } \quad \operatorname{Gr}^{0} R(T)_{\mathbb{Q}}=\mathbb{Q} .
$$

For this, let $i_{w}: S \rightarrow G / B$ denote the $S$-valued point $w B / B$ of $G / B$ for $w \in W$. Then, by the equivariant excess intersection formula (3.8), we have

$$
i_{w}^{*}\left(i_{e}\right)_{*}(1)= \begin{cases}0 & \text { for } \quad w \neq e \\ \prod_{\alpha \in R_{+}}\left(1-e^{\alpha}\right) & \text { for } \quad w=e\end{cases}
$$

since the class of the conormal sheaf $\mathcal{N}_{i_{e}}$ of $i_{e}$ equals $\sum_{\alpha \in R_{+}} e^{\alpha}$ by formula (4) of [J] on p. 229 . Thus, the class $\left(i_{e}\right)_{*}(1)$ of the structure sheaf of the $T$-fixed point $e B / B$ is contained in the ideal $F_{0}^{l\left(w_{0}\right)}(T, G / B)$ (alternatively, this follows from Theorem (5.10)(a) like in Remark $(5.13))$, and we certainly have $\pi_{*}\left(i_{e}\right)_{*}(1)=1$. Therefore, it suffices to prove the formula above with $\psi^{w_{0}}$ replaced by $\left(i_{e}\right)_{*}(1)$. But by definition of $L$, we have $L\left(\overline{\left(i_{e}\right)_{*}(1)}\right)=1$ (note that $\left.c_{1}\left(e^{-\alpha}\right)=\left(1-e^{\alpha}\right)+I^{2}\right)$. This completes the proof of Theorem (7.11).

(7.12) Example. Similarly to the formal proof of the Grothendieck-Riemann-Roch theorem used for the proof above, there is the following formal proof for the equivariant Adams-RiemannRoch theorem (4.5) using the operator $D$ : Let $j \in \mathbb{N}$ and $f \in R(T)$. Then we have:

$$
\begin{aligned}
\psi^{j} D & (\phi(1 \otimes f))=\psi^{j} \sum_{w \in W}\left(f \cdot \prod_{\alpha \in R_{+}} \frac{1}{1-e^{\alpha}}\right)^{w} \\
= & \sum_{w \in W}\left(\psi^{j} f \cdot \prod_{\alpha \in R_{+}} \frac{1}{1-e^{j \alpha}}\right)^{w} \\
= & \sum_{w \in W}\left(\psi^{j} f \cdot \prod_{\alpha \in R_{+}} \frac{1-e^{\alpha}}{1-e^{j \alpha}} \cdot \prod_{\alpha \in R_{+}} \frac{1}{1-e^{\alpha}}\right)^{w} \\
= & D\left(\psi^{j} \phi(1 \otimes f) \cdot \theta^{j}\left(T_{\pi}^{\vee}\right)^{-1}\right) .
\end{aligned}
$$




\section{References}

[At] M. F. AtтYAн, Characters and cohomology of finite groups, Publ. Math. IHES 9 (1961), 23-64.

[AT] M. F. AtiYah and D. O. TALL, Group representations, $\lambda$-rings and the $J$ homomorphism, Topology 8 (1969), 253-297.

[Ba] H. BAss, "Algebraic K-theory", Math. Lecture Note Series (Benjamin, New York, 1968).

[SGA6] P. Berthelot, A. Grothendieck and L. Illusie, "Théorie des Intersections et Théorème de Riemann-Roch", Lecture Notes in Math. 225 (Springer, New York, 1971).

[Bo] R. BoltJe, "Mackey functors and related structures in representation theory and number theory", Report No. 327 (Institut für Mathematik der Universität Augsburg, 1995).

[BV] M. Brion and M. Vergne, An equivariant Riemann-Roch theorem for complete, simplicial toric varieties, J. Reine Angew. Math. 482 (1997), 67-92.

[BC] D. Burns and T. Chinburg, Adams operations and integral Hermitian-Galois representations, Amer. J. Math. 118 (1996), 925-962.

[CNT] Ph. Cassou-Noguès and M. J. Taylor, Opérations d'Adams et Groupe des classes d'Algèbre de groupe, J. Algebra 95 (1985), 125-152.

[CEPT] T. Chinburg, B. Erez, G. Pappas and M. J. Taylor, Riemann-Roch type theorems for arithmetic schemes with a finite group action, J. Reine Angew. Math. 489 (1997), 151-187.

[CR] C. W. Curtis and I. Reiner, "Methods of representation theory with applications to finite groups and orders", vol. I, Pure Appl. Math. (Wiley, New York, 1981).

[De] M. Demazure, Désingularisation des variétés de Schubert généralisées, Ann. Sci. École Norm. Sup. (4) 7 (1974), 53-88.

[SGA3] M. Demazure and A. Grothendieck, "Schémas en Groupes I, II, III", Lecture Notes in Math. 151, 152, 153 (Springer, New York, 1970).

[EG] D. EDIDIN and W. Graham, Equivariant intersection theory, preprint (alggeom/9603008).

[FH] W. Fulton and J. Harris, "Representation theory", Grad. Texts in Math. 129 (Springer, New York, 1991).

[FL] W. Fulton and S. LAng, "Riemann-Roch algebra", Grundlehren Math. Wiss. 277 (Springer, New York, 1985).

[FM] W. Fulton and R. MacPherson, Characteristic classes of direct image bundles for covering maps, Ann. of Math. 125 (1987), 1-92.

[Gr] D. R. Grayson, Exterior power operations on higher $K$-theory, K-Theory 3 (1989), 247-260. 
[Gro1] A. Grothendieck, Sur quelques propriétés fondamentales en théorie des intersections, Seminaire C. Chevalley, $2^{e}$ année, "Anneaux de Chow et applications" (Secr. Math. Paris, 1958).

[Gro2] A. Grothendieck, Classes de Chern et représentations linéaires des groupes discrets, in J. Giraud et al., "Dix exposés sur la cohomologie des schémas", Adv. Stud. Pure Math. (North-Holland Publishing Company, Amsterdam, 1968), 215-305.

[EGA] A. Grothendieck and J. A. Dieudonné, "Eléments de Géométrie Algébrique I", Grundlehren Math. Wiss. 166 (Springer, New York, 1971); “... II, III”, Publ. Math. IHES 8 (1961), 11 (1961), 17 (1963).

[Ha] R. Hartshorne, "Algebraic geometry", Graduate Texts in Math. 52 (Springer, New York, 1977).

[Hi] H. L. Hiller, $\lambda$-rings and algebraic $K$-theory, J. Pure Appl. Algebra 20 (1981), 241-266.

[J] J. C. Jantzen, "Representations of algebraic groups", Pure Appl. Math. 131 (Academic Press, Boston, 1987).

[Ke] M. Kervaire, Opérations d'Adams en Théorie des représentations linéaires des groupes finis, Enseign. Math. 22 (1976), 1-28.

[Ko0] B. KÖck, "Das Lefschetz- und Riemann-Roch-Theorem in der höheren äquivarianten K-Theorie", Dissertation (Regensburg, 1989).

[Ko1] B. Köck, Chow motif and higher Chow theory of $G / P$, Manuscripta Math. 70 (1991), 363-372.

[Ko2] B. Köck, Das Adams-Riemann-Roch-Theorem in der höheren äquivarianten $K$ Theorie, J. Reine Angew. Math. 421 (1991), 189-217.

[Ko3] B. Köck, The Lefschetz theorem in higher equivariant $K$-theory, Comm. Algebra 19 (1991), 3411-3422.

[Ko4] B. KÖCK, Higher $K^{\prime}$-groups of integral group rings, $K$-Theory 4 (1991), 177-187.

[Ko5] B. KÖck, Shuffle products in higher K-theory, J. Pure Appl. Algebra 92 (1994), 269307.

[Ko6] B. KöcK, "The Grothendieck-Riemann-Roch theorem in the higher $K$-theory of group scheme actions", Habilitationsschrift (Karlsruhe, 1995).

[Ko7] B. Köck, On Adams operations on the higher $K$-theory of group rings, in: G. BANASZAK et al. (eds.), "Algebraic $K$-theory (Poznań, 1995)", Contemp. Math. 199 (Amer. Math. Soc., Providence, 1996), 139-150.

[Ko8] B. Köck, Adams operations for projective modules over group rings, Math. Proc. Cambridge Philos. Soc. 122 (1997), 55-71.

[Ko9] B. Köck, Operations on locally free classgroups, preprint (Karlsruhe, 1997).

[Ko10] B. KöcK, A Riemann-Roch formula for tensor power representations of the symmetric group, in preparation. 
[KK1] B. Kostant and S. Kumar, The nil Hecke ring and cohomology of $G / P$ for a KacMoody group G, Adv. Math. 62 (1986), 187-237.

[KK2] B. Kostant and S. Kumar, $T$-equivariant $K$-theory of generalized flag varieties, $J$. Differential Geom. 32 (1990), 549-603.

[Kr] Ch. Kratzer, $\lambda$-Structure en $K$-théorie algébrique, Comment. Math. Helv. 55 (1980), 233-254.

[La] A. Lascoux, Anneau de Grothendieck de la variété de drapeaux, in "The Grothendieck Festschrift, vol. III", Progr. Math. 88 (Birkhäuser, Boston, 1990), 1-34.

[Le] H. W. Lenstra, Grothendieck groups of abelian group rings, J. Pure Appl. Algebra 20 (1981), 173-193.

[Man] Y. I. Manin, Lectures on the $K$-functor in algebraic geometry, Russian Math. Surveys 24, No. 5 (1969), 1-89.

[Mo] R. Morelli, The $K$ theory of a toric variety, Adv. Math. 100 (1993), 154-182.

[Mum] D. Mumford, "Geometric invariant theory", Ergeb. Math. Grenzgeb. 34 (Springer, New York, 1965).

[Q] D. Quillen, Higher algebraic $K$-theory: I, in H. BAss (ed.), "Algebraic $K$-Theory I (Seattle, 1972)", Lecture Notes in Math. 341 (Springer, New York, 1973), 85-147.

[Sn] V. P. Snaith, Invariants of representations, in J. F. Jardine and V. P. Snaith (eds.), "Algebraic $K$-theory: Connections with geometry and topology (Lake Louise, 1987)", NATO Adv. Sci. Inst. Ser. C: Math. Phys. Sci. 279 (Kluwer Acad. Publ., Dordrecht, 1989), 445-508.

[So] C. Soulé, Opérations en K-Théorie algébrique, Canad. J. Math. 37, No. 3 (1985), 488-550.

[Su] H. Suminiro, Equivariant completion II, J. Math. Kyoto Univ. 15 (1975), 573-605.

[Ta] G. Tamme, The theorem of Riemann-Roch, in M. Rapoport, N. Schappacher and P. Schneider (eds.), "Beilinson's conjectures on special values of $L$-functions", Perspect. in Math. 4 (Academic Press, Boston, 1988), 103-168.

[Tho] C. B. Thomas, "Characteristic classes and the cohomology of finite groups", Cambridge Stud. Adv. Math. 9 (Cambridge Univ. Press, Cambridge, 1986).

[Th1] R. W. Thomason, Absolute cohomological purity, Bull. Soc. Math. France 112 (1984), 397-406.

[Th2] R. W. Thomason, Lefschetz-Riemann-Roch theorem and coherent trace formula, Invent. Math. 85 (1986), 515-543.

[Th3] R. W. Thomason, Algebraic $K$-theory of group scheme actions, in W. Browder (ed.), "Algebraic topology and algebraic $K$-theory (Princeton, 1983)", Ann. Math. Stud. 113, (Univ. Press, Princeton, 1987), 539-563.

[Th4] R. W. Thomason, Une formule de Lefschetz en $K$-théorie équivariante algébrique, Duke Math. J. 68 (1992), 447-462. 
[Th5] R. W. Thomason, Les $K$-groupes d'un schéma éclaté et une formule d'intersection excédentaire, Invent. Math. 112 (1993), 195-215.

[We] C. A. Weibel, Homotopy algebraic $K$-theory, in M. R. Stein and R. K. Dennis (eds.), "Algebraic $K$-theory and algebraic number theory (Honolulu, 1987)", Contemp. Math. 83 (Amer. Math. Soc., Providence, 1989), 461-488. 